\title{
Barotropic wind-driven circulation patterns in a closed rectangular basin of variable depth influenced by a peninsula or an island
}

\author{
B. V. Chubarenko ${ }^{1,2}$, Y. Wang ${ }^{1}$, I. P. Chubarenko ${ }^{1,2}$, K. Hutter ${ }^{1}$ \\ ${ }^{1}$ Institute of Mechanics, Darmstadt University of Technology, Hochschulstr. 1, D-64289 Darmstadt, Germany \\ ${ }^{2}$ Laboratory of Coastal Systems Study, Atlantic Branch of P.P. Shirshov Institute of Oceanology of Russian Academy of Science, \\ Prospect Mira, 1, 236000, Kaliningrad, Russia
}

Received: 28 September 1999 / Revised: 13 March 2000 / Accepted: 3 April 2000

\begin{abstract}
We study how a coastal obstruction (peninsula or coastal island) affects the three-dimensional barotropic currents in an oblong rectangular basin with variable bathymetry across the basin width. The transverse depth profile is asymmetric and the peninsula or island lies in the middle of the long side of the rectangle. A semi-spectral model for the Boussinesq-approximated shallow water equations, developed in Haidvogel et al. and altered for semi-implicit numerical integration in time in Wang and Hutter, is used to find the steady barotropic state circulation pattern to external winds. The structural (qualitative) rearrangements and quanti2tative features of the current pattern are studied under four principal wind directions and different lengths of the peninsula and its inclination relative to the shore. The essentially non-linear relationships of the water flux between the two sub-basins (formed by the obstructing peninsula) and the corresponding crosssectional area left open are found and analysed. It is further analysed whether the depth-integrated model, usually adopted by others, is meaningful when applied to the water exchange problems. The flow through the channel narrowing is quantitatively estimated and compared with the three-dimensional results. The dynamics of the vortex structure and the identification of the upwelling/down-welling zones around the obstruction are discussed in detail. The influence of the transformation of the peninsula into a coastal island on the global basin circulation is considered as are the currents in the channel. The geometric and physical reasons for the anisotropy of the current structure which prevail through all obtained solutions are also discussed.
\end{abstract}

Key words: Oceanography: general (limnology; numerical modeling) - Oceanography: physical (currents)

Correspondence to: Y. Wang

e-mail: wang@mechanik.tu-darmstadt.de

Dedicated to Professor L.A. Mysak on the occasion of his sixtieth birthday.

\section{Introduction}

The dynamics of fjords, lakes and lagoons differ in many respects from those of the ocean; shoreline obstructions affect the fluid dynamics not only in details, but often even in their global structure, e.g. in the water exchange mechanisms between parts of the entire basin. When this happens these elements can no longer be considered as negligibly small, regardless of their size, in comparison to the spatial scales of the basin.

In spite of a large variety of shapes of the coast lines of natural closed and semi-closed water basins like lakes, lagoons, fjords and estuaries, a peninsula or a nearshore island may constitute the main structural element of the landscape. Some typical examples of coastal line configurations are shown in Fig. 1, where we collected a group of well known large lakes (Bartholomew, 19551959). For example, Lake Balaton (Hungary), Lake Balkhash (Kazakhstan), Lake Erie, Lake Superior, Lake Winnipeg (all Canada/USA) and Lake Vanern (Sweden) have oblong isolated peninsulas with varied inclinations to the coast line. Lake Baikal (Russia), Lake Chudskoe (Russia/Estonia), the Dead Sea (Israel), Lake Daryacheh/Urmia (Iran), Lake Tanganyika (Burundi/Tanzania/Zaire/Zambia) have significant headlands which also divide their areas into various sub-basins. Near-coastal islands play a role in bordering sub-basins in Lakes Baikal (Russia), Constance (Germany/Switzerland), Vanern (Sweden), in Neusiedler See (Austria/Hungary) and Lago Iseo (Northern Italy). There are obviously many more, and these only serve as examples.

In all the examples either a peninsula or an island constitute a visible obstruction in plan view, which narrows the lake width or area at least locally and thus influences not only the local current patterns and processes (see Kocsis et al., 1998, and the list of references therein) but may affect the circulation as a whole and thus give rise to water exchange in the areas that are typical for the presence of these obstructions. When the sub-basins separated by the obstruction are 

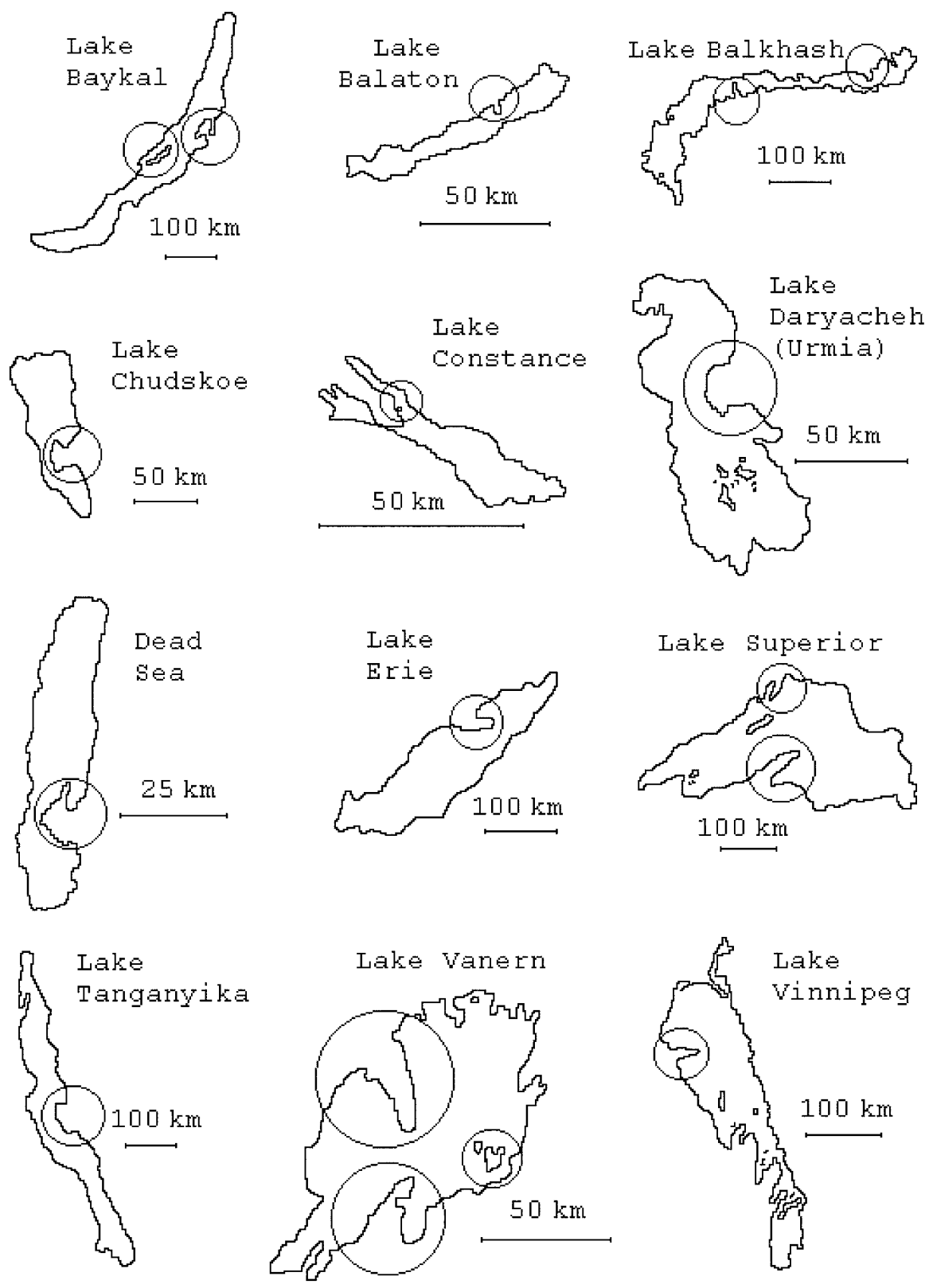

very different from one another with regard to their sources and sinks along the boundaries (such as inlet and outlet rivers, artificial inlets, straits to adjacent basins, etc.), the water exchange processes between these sub-basins is a key factor in defining the temporal and spatial variations of the physical and biochemical environment for the ecosystem of the basin as a whole. In addition, all transport mechanisms of nutrients and pollutants are chiefly governed by advection and vertical diffusion enhanced by narrows; so it is obvious that the presence of such obstructions is extremely important for the behaviour of the lake water system.

Our aim is to study how and when a peninsula or island will affect the overall circulation of a lake that is subjected to external wind forcing. We shall do this here for barotropic currents using a fully three-dimensional hydrodynamic model. In particular, it is our goal to describe the characteristic features of the structure of the wind-induced current and the associated water exchange between the sub-basins in accordance with the existence, size and orientation of the peninsula or near-coastal island. Idealised geometry of the basin, peninsula and island will be presumed and simple steady uniform wind scenarios in the principal directions of the basin will be employed and followed until steady state current patterns will have been established. With $L, H$ being typical horizontal and vertical length scales and $g$ the gravity constant, a typical barotropic time scale for barotropic transients is $T=L /(g H)^{1 / 2}$. For lakes and lagoons of typical extent (say $10-500 \mathrm{~km}$ length) these times are one to a few hours, so the statement of the problem is reasonable. It is expected that the circulation patterns will disclose typical features that can be associated with the existence of the obstructions and thus can be generalised to other basins, exhibiting similar features. 
The applied aspect of our study is to gain an understanding for the limnological processes, of the near shore zone of a lake, here the vicinity of the island Mainau in the Überlinger See, a long isolated arm of Lake Constance. Lake Constance is a glacial Alpine lake of which the physical limnological aspects, and in particular the wind-induced current patterns have been thoroughly studied in the last 20 to 30 years, see Bäuerle (1994), Bäuerle et al. (1998), Heinz et al. (1990), Hollan and Simons (1978), Hollan et al. (1990), Hutter et al. (1998), Hutter and Wang (1998), Kocsis et al. (1998), and Wang and Hutter (2000). However, all these studies are mostly addressed to the global basin-scale behaviour, dismissing the littoral zone because in these studies it was basically of subgrid scale. The method of substructuring needed to be developed in order that the detailed processes in the smaller scale regions could be scrutinised.

A numerical code SPEM is used which allows computation of the three-dimensional currents in barotropic and baroclinic situations if the basic vertical density profile is prescribed. We restrict attention to uniform density to model typical late autumn, winter and early spring situations, baroclinic situations will be studied later. This computational approach is advantageous, because direct measurements cannot provide a complete picture regarding the variations of the exchange parameters through space and time under different forcing factors. Furthermore, such numerical methods are likely the only ones by which the circulation pattern in a lake can be typified as a function of the relative size of the peninsula.

The simplest approach was taken using a rectangular basin with asymmetric bathymetry across the width. The long ends are in the west and the east of the basin, the long shores in the south and north, and the peninsula or island is positioned in the centre of the southern shore with its axis perpendicular to the shore or at an angle different from $90^{\circ}$ pointing towards west or east. On the $f$-plane these orientations are insignificant, used here merely to easily identify the orientations and positions of the obstructions, wind and currents, respectively.

\section{Study area and model specifications}

Two criteria were decisive in choosing the shape and bathymetry of the basin for simulations. It should first represent the main features of natural lakes but still be simple enough to permit rapid numerical discretisation. It should isolate the processes due to the presence of the peninsula or near-coastal island; i.e. lateral boundaries of the main basin should possibly not disturb the current structure induced by the peninsula or the island so that the role played by these obstructions could be identified, in particular with regard to the limitation of the water exchange processes between the sub-basins. The peninsula was positioned in the middle of the southern shore (Fig. 2a). Its length and shape were varied giving rise to a variable cross section $\left(S_{\min }\right)$ in the middle of the lake and, thus, leading to a variable passage between the two sub-basins as the size of the peninsula was varied.

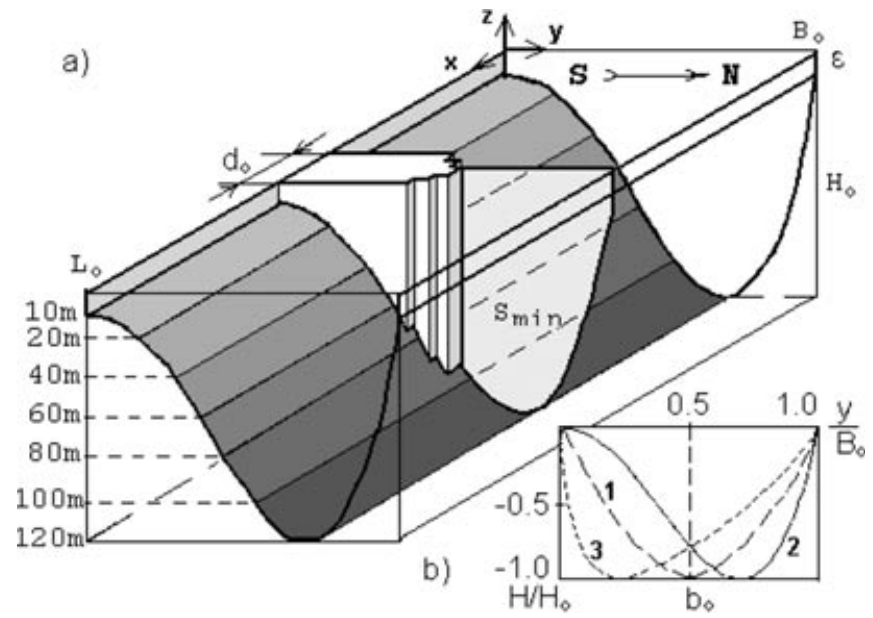

Fig. 2. a Perspective view of a rectangular basin with variable cross section but constant profile in its long direction. Cartesian co-ordinate system; $x$ in the long direction, $y$ transverse and $z$ upwards against gravity. Origin in the southwest corner on the free surface. Dimensions are $L_{0}=15 \mathrm{~km}, B_{0}=4 \mathrm{~km}$ and $H_{0}=120 \mathrm{~m}$. In the middle of the southern shore a peninsula of width $d_{0}=1230 \mathrm{~m}$. The cross section from the tip of this peninsula to the opposite shore has area $S_{\min }$. b Bathymetric profiles according to formula (1) for three different values of the exponent $b:--b=1,-b=1.94$ (typical for $b>1$ ) and $-\cdots-b=0.7$ (typical for $0<b<1$ )

The transverse bathymetric profile was chosen to be given by (for the coordinate system see Fig. 2a)

$\frac{H(x, y)}{H_{0}}=1-4\left[\left(\frac{y}{B_{0}}\right)^{b}-0.5\right]^{2}+\varepsilon$,

in which $b$ is a parameter by which the profile can be varied. Note that for $\varepsilon=0$ and $y=0$ or $y=B_{0}$ the depth is zero. Our code does not permit zero water depth at the shoreline which is the reason why we chose a set-off parameter $\varepsilon$. The exponent $b$ allows the shape to be varied (see Fig. 2b). For $b=1$ a symmetric, quadratic parabola is obtained, $0<b<1$ corresponds to a trough of which the deepest point $b_{0}$ is in the interval $0<y<B_{0} / 2$ and whose slope at $y=0$ is infinite; $b>1$ on the other hand, yields a depth profile whose minimum lies in the interval $B_{0} / 2<y<B_{0}$ and which approaches the origin at $y=0$ with the slope $\partial H / \partial y(y=0)=0$; in the vicinity of the southern shore this profile possesses a convex shape and is therefore particularly useful for the study of the littoral zones. We have used $b=1.94$, $l_{0} / B_{0}=0.7$. As long as $H_{0}$ is constant the bathymetric level lines are straight lines parallel to the $x$-axis. It could be taken to be $x$-dependent, but we choose $H_{0}=$ const to isolate the significance of the peninsula (Kocsis et al., 1998). Finally we choose $H_{0}=120 \mathrm{~m}$ (Bäuerle et al., 1998), to be much larger than typical pycnocline depths of natural lakes, fjords and straits. The horizontal extent of the study area is $15 \times 4 \mathrm{~km}^{2}$, typical for an Alpine lake. The width of the peninsula is taken to be $1230 \mathrm{~m}$, corresponding to six grid size lengths; it is sufficiently large to represent the real size of natural coastal variations and sufficiently narrow to avoid (in our case) the influence of the lateral boundaries on the current 
pattern around the peninsula. The length of the peninsula varies from two grid size widths (of $160 \mathrm{~m}$ each) to 20 , thus covering up to $83.3 \%$ of the total width of the basin. The boundary of the peninsula and the near-shore island were defined by the grid (for simplicity) with a shore depth corresponding to the depth defined by Eq. (1). Since the code uses linear interpolation from grid point to grid point this is tantamount to a shore slope around the peninsula defined by the local depth and the mesh size. In our computations we chose the Coriolis parameter $f=1.07 \times 10^{-4} \mathrm{~s}^{-1}$, corresponding to the geographic latitude of $43^{\circ}$ for Alpine lakes. Because our aim is modelling Alpine lakes the variability of $f$ is not very important.

\section{Methods}

The balance laws of mass and momentum form the hydrodynamic field equations for the considered fluid system. We apply these hydrodynamic equations in the shallow water approximation, with the Coriolis term and the hydrostatic pressure assumption implemented. Diffusivities are taken into account by differentiating between the horizontal and vertical directions. By doing so the anisotropy effects of the turbulent intensity are accordingly considered.

To solve the system of differential equations numerically, a semi-spectral model was designed with semi-implicit integration in time. The model is the semispectral model SPEM developed by Haidvogel et al. (1991), as extended by Wang and Hutter (1998) to account for implicit temporal integration: because of the small size of lakes in comparison to the ocean the original SPEM model designed for ocean problems had to be altered to permit economically justifiable time steps in the computation of the circulation within the lake.

In Wang and Hutter (1998) several finite difference schemes, implicit in time, were introduced; that scheme which uses implicit integration in time for the viscous terms in the vertical direction alone was the most successful one. In this semi-spectral model the variables in the vertical direction are accounted for by a superposition of Chebyshev polynomials, but finite difference discretisation is used in the horizontal direction. The model employs prior to its discretisation a $\sigma$-transformation and thus maps the lake domain with variable depth to a new domain with constant depth.

SPEM uses zeroth order turbulent closure and allows for algebraic relationships of the horizontal and vertical diffusivities or, simpler, employs prescribed diffusivities as functions of space and time. This was our choice; it was assumed that a well-mixed upper layer was formed under the imposed wind stress giving rise to wind waves and Langmuir circulation and thus efficient mixing. The vertical turbulent viscosity coefficient was vertically varied according to the formula

$$
v_{v}=\frac{v_{\text {top }}+v_{\text {bot }}}{2}+\frac{v_{\text {top }}-v_{\text {bot }}}{2} \arctan \left[k z_{0}\left(1-\frac{z}{z_{0}}\right)\right] \text {, }
$$

in which $z$ is the vertical co-ordinate, $v_{\text {top }}$ and $v_{\text {bot }}$ are reminiscent for a value of an upper and lower layer vertical diffusivity, $z_{0}$ is the location of the inflexion point of the profile $v_{v}(z)$, and $k$ is a constant, which is responsible for the steepness of the transition from large, upper layer values to small, hypolimnion values of $v_{v}$; the larger $k$ is, the sharper will be the "turbocline", i.e. the smaller will be the transition zone between the upper, well mixed zone and the deep water zone. Examples are given in Fig. 3.

Values for the diffusivities were taken as $v_{\text {top }}=0.015 \mathrm{~m}^{2} \mathrm{~s}^{-1}$ and $v_{\text {bot }}=0.005 \mathrm{~m}^{2} \mathrm{~s}^{-1} \quad(k=100$ and $\left.z_{0}=15\right)$. Their choice is not entirely free as their values affect the numerical stability of the integration using the semi-implicit code SPEM. These values guarantee stability for the selections $\Delta x=205 \mathrm{~m}$, $\Delta y=160 \mathrm{~m}, \Delta t=15 \mathrm{~min}$ and a choice of 20 Chebychev polynomials in the vertical discretisation, but they are slightly larger than typical turbulent kinematic viscosities suggested by measurements (Hutter, 1984). This increased dissipation will slightly reduce the maximum values of the current velocities and will also dampen the oscillations induced by the transient wind input, see Hutter and Wang (1998) and Wang and Hutter (1998); however, since steady state values are of interest, this is no serious drawback, because the steady current behaviour is only slightly affected by it. The most significant influence may be the thickness of the Ekman layer close to the surface.

In principle the horizontal diffusivities do also vary with position, but such variations are poorly known and depend on the surrounding landforms, the fetch of the wind and other parameters. Computations have indicated that the values of the horizontal diffusivities are not very critical, and a constant value of $1 \mathrm{~m}^{2} \mathrm{~s}^{-1}$ is standard.

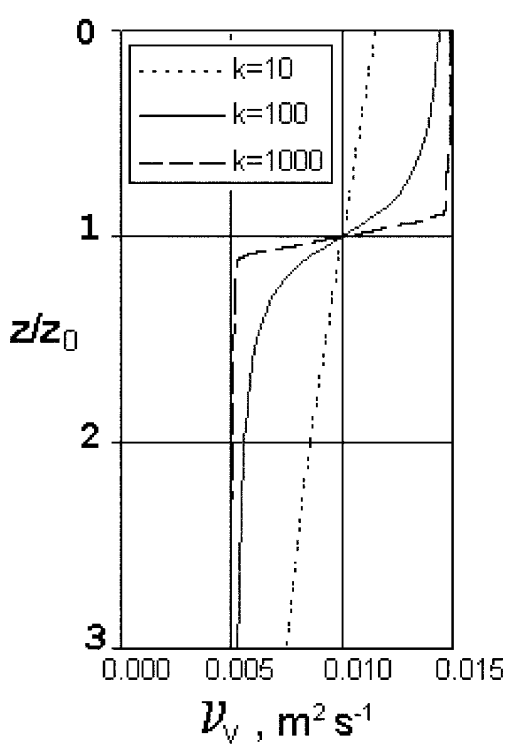

Fig. 3. Kinematic turbulent viscosities as obtained with Eq. (2) using the following parameters: $v_{\text {top }}=0.015 \mathrm{~m}^{2} \mathrm{~s}^{-1}, v_{\text {bot }}=0.005 \mathrm{~m}^{2} \mathrm{~s}^{-1}$, $z_{0}=15$ and $k$ as given in the inset 
This numerical code has proved its suitability in several lake applications, including diffusion problems (Hutter and Wang, 1998), substructuring procedures (Wang and Hutter, 2000) and wave dynamics in barotropic and baroclinic wind-driven circulation dynamics (Hutter et al., 1998). For this reason we refrain from presenting the governing equations and directly pass on to the application of this code to the barotropic motion.

\section{Results}

\subsection{General current patterns}

Five series of simulations were executed for the above described basin which had the following different configurations of peninsula and island:

a. Basic reference configuration without any obstacles (peninsula or island) inside the basin, pure rectangular basin

b. Series of 10 peninsulas of various lengths with axes perpendicular to the shore, each under four main wind directions

c. Near-shore island with the axis perpendicular to the shore, separated from the coast by a narrow channel under four main wind directions

d. Two versions of a fixed length peninsula inclined to the west and to the east under four main wind directions

e. Two inclined islands separated from the coast by a narrow channel under four main wind directions

The four three-dimensional wind driven current patterns were simulated for each configuration. Spatially uniform winds, Heaviside in time, in the principal directions of the rectangle were applied. Computations were commenced with zero wind and currents starting from rest and followed until steady conditions were reached. This was the case after three days of computation of the model time, as already demonstrated in Wang and Hutter (2000).

Results are diagrammatically displayed in Figs. 4-7, both for a rectangular basin without a peninsula or island and with a peninsula in the middle of the southern shore. The figures show the current patterns in horizontal plane levels at the free surface and at 10, 20, 40, 60 and $80 \mathrm{~m}$ depth. As would be expected for the unobstructed rectangle subjected to wind in the long direction, there are coastal jets along the northern and southern shore directed with the wind (see Figs. 4, 5); these currents are larger in the vicinity of the southern shore and more widely distributed than along the northern shore, this is because of the shallower shore in the southern than in the northern region. The surface current in the lake interior is basically across the width, again as would be expected in a rotating channel. Moving down to larger depths, a return current is established in the middle of the rectangular basin. In addition, for west winds a cyclonic gyre is formed at the eastern end which embraces the entire depth of the basin in this region (Figs. 4, 8). For the same wind from the east (Figs. 5, 8) essentially the same current structure is established but with opposite direction. The coastal jet along the southern shore is wider, with larger velocities than for west winds. The two gyres, now anti-cyclonically rotating, are less pronounced, wider than for west winds and do not embrace the entire depth. Special supplementary simulations disclose the following additional results: if the long ends of the rectangle are not be blocked by vertical walls, but replaced by a smooth transition of the bathymetry to zero depth, the gyres would also form for winds in the long direction. Moreover, the wind-driven current structure in a rectangular basin with constant depth bounded by vertical walls contains no gyres. These results can, in large parts, be qualitatively interpreted in terms of simple circulation patterns of a rotating closed hydrodynamic system. For instance, the strong coastal jet can e.g. be explained by using steady state mass and momentum balances in a (non-rotating) constant cross section channel. It yields the along channel volume flux $U$ with a transverse variation proportional to $\left(H_{\text {mean }}-H\right)$, where $H$ and $H_{\text {mean }}$ are, respectively, the water depth and its crosssectional mean (see Csanady, 1984), and they explain the large coastal currents and the wider extent of them where the shallow depth extends farther away from the shore, as evidenced in Fig. 2. The counter-clockwise rotation of the off-shore current with depth, on the other hand, is in line with the Ekman spiral (Heaps, 1984; Csanady, 1984). Its rotation below approximately $40 \mathrm{~m}$ ceases, because due to the finiteness of the basin, mass balance requires a return current (Heaps, 1984; Wang and Hutter, 1998). This steady return current is topographically guided and must be on the $f$-plane along the isobaths as can be seen from the rigid-lid restricted topographic wave equation

$\nabla \cdot\left(\frac{1}{H} \nabla \frac{\partial \psi}{\partial t}\right)+\mathscr{J}\left[\psi, \frac{f}{H}\right]=0$

where $\psi$ is the volume-flux-transport stream function, $\nabla=(\partial / \partial x, \partial / \partial y), \mathscr{J}[\bullet, \bullet]$ is the Jacobian operator (Stocker and Hutter, 1987). The topographic wave Eq. (3) also explains the absence of gyres in a constant depth basin because under such conditions $\psi=$ const is the only solution of Eq. (3). The asymmetric formation of the gyres at the eastern end only, is due to the transverse asymmetry of the bathymetric profile (a symmetric profile generates gyres at both ends). It is not a bay mode response due to longitudinal variations of the bathymetry (Stocker and Hutter, 1987), for if it were, differences of gyre geometries and strengths should be discernable for computations with vertical and shoaling channel ends, but they are not. On the other hand, with the bathymetric profile of Fig. 2 the topographic restoring forces for a given transverse current are strong at the SE corner and weak in the NW corner, making the existence of strong gyres at the eastern end more likely than at the western end. Furthermore, differences in strength and size of the gyres formed under west and east winds are in full 


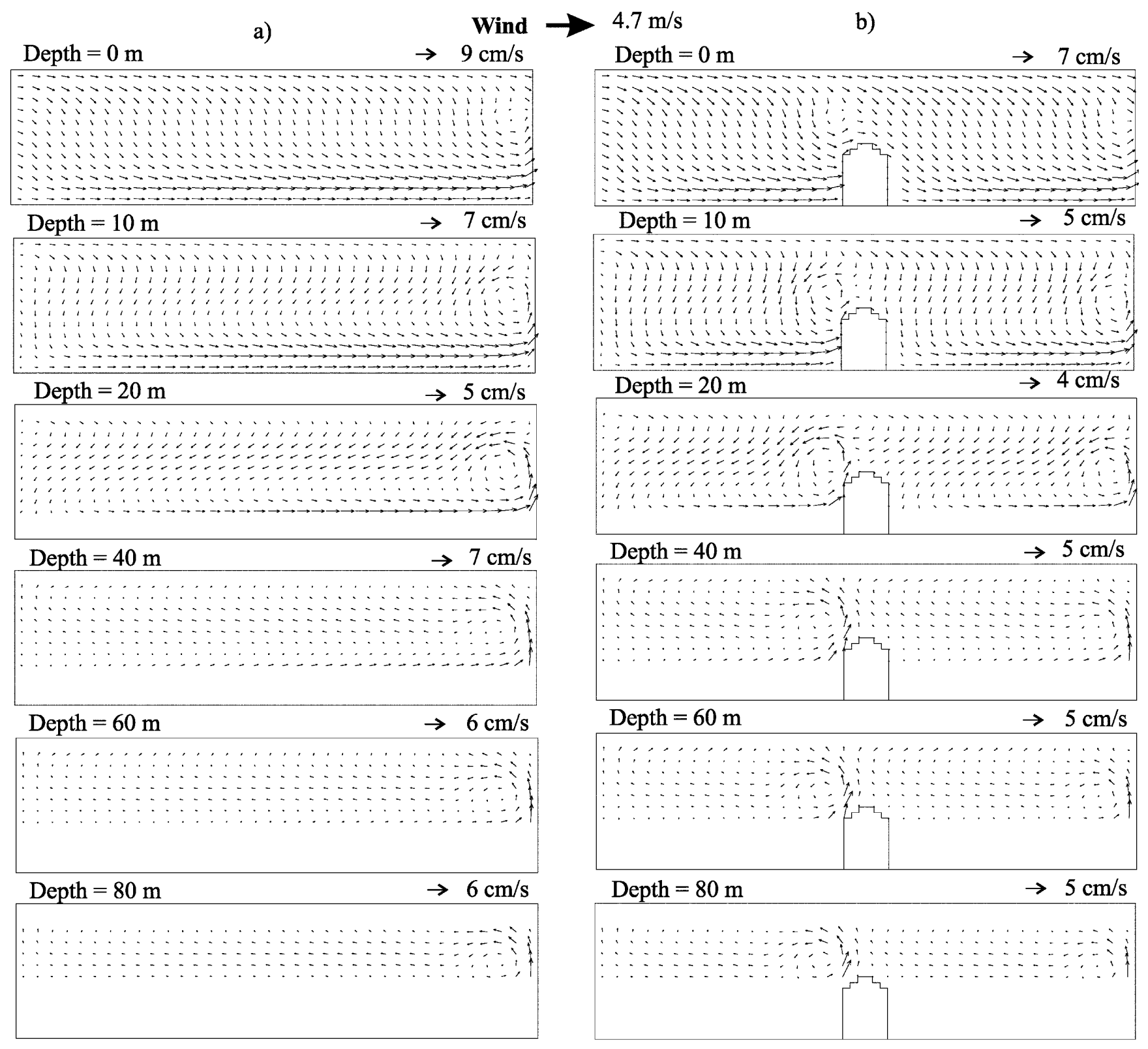

Fig. 4a, b. Vector plots of the horizontal velocity for steady conditions, three days after the onset of a spatially uniform wind of $4.7 \mathrm{~m} \mathrm{~s}^{-1}$ (corresponding to a wind shear stress of $0.05 \mathrm{~Pa}$ ) from west at the depths $0,10,20,40,60$ and $80 \mathrm{~m}$ below the free surface; the

panels on the left $\mathbf{a}$ are for a rectangular basin with bathymetric profile as shown by the line 2 in Fig. 2 , right $\mathbf{b}$ for a rectangle with a peninsula of more than critical length

agreement with the general geophysical rule: cyclonically rotating gyres (i.e. gyres rotating in the same direction as the Earth, counter-clockwise in the Northern Hemisphere) are supported and localised by the Coriolis force, while anticyclonically rotating ones are smoothed and spread (Gill, 1982).

The surface currents for a wind from north (Fig. 6) and south (Fig. 7) are set off to the right of the wind by approximately $45^{\circ}$ almost everywhere except close to the leeward shore where they are directed with the wind. As one moves down to larger depths these currents rotate clockwise down to $40 \mathrm{~m}$, after which they point in the long direction of the basin to the left of the wind, corresponding to the geostrophic balance established

with the wind set-up in the north-south direction at the surface. This rotation into the along-channel flow at depth is faster and more direct than for the cases with winds from $\mathrm{E}$ and $\mathrm{W}$, and it manifests itself here as a true flow locking at depth. The reason is that the flow at depth is supported here by two processes, the superposed geostrophic and topographic balance, whilst for $\mathrm{E}$ - and $\mathrm{W}$-winds it is only the latter that forces the flow into the along-channel direction. This may also be the cause that no gyre develops for winds from north and south whereas they do for winds from west and east. Indeed, for winds in the long direction the initial (transient) flow is topographically guided, but the transients of the Ekman drift that is established and 


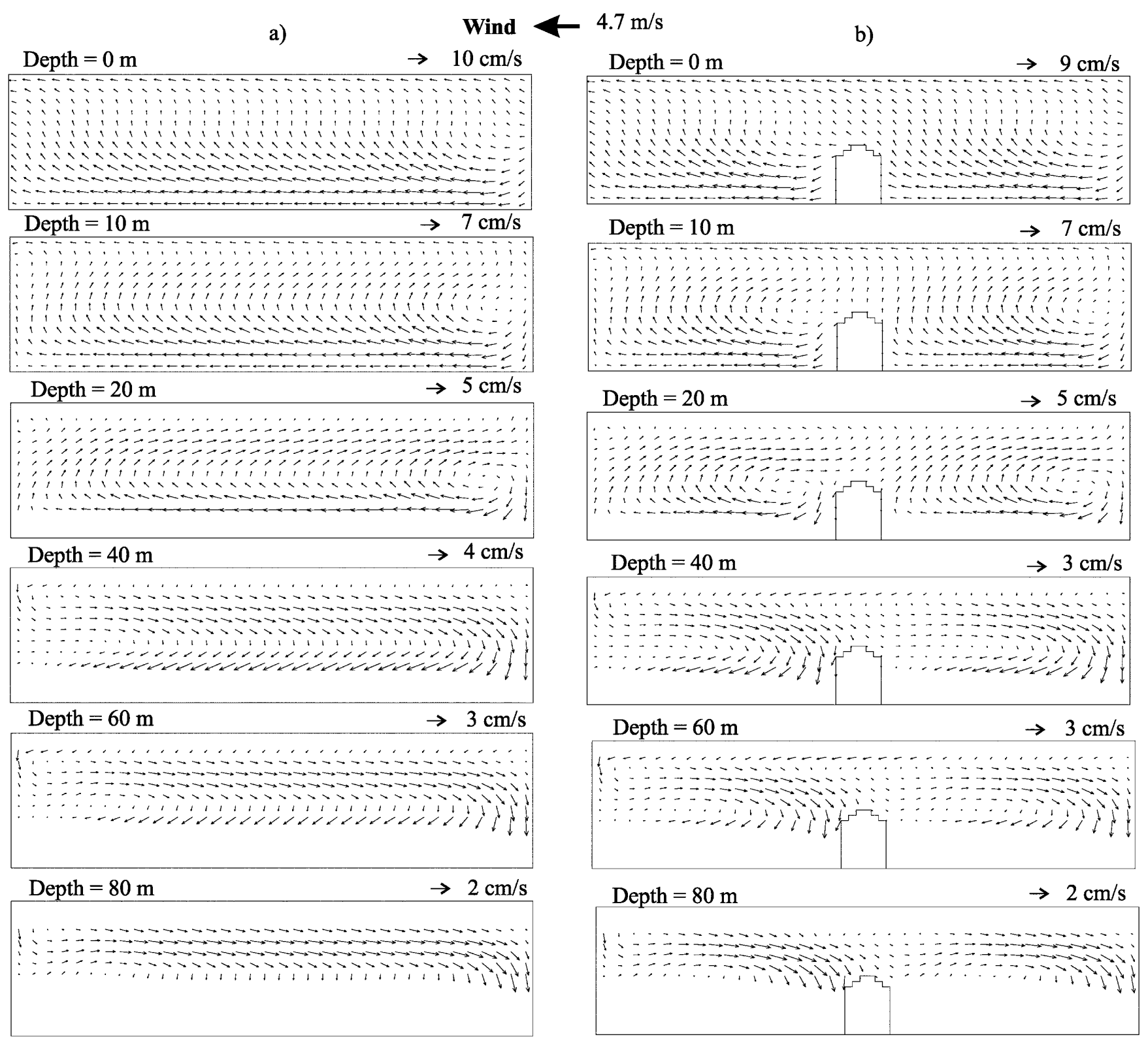

Fig. 5a, b. Same as Fig. 4, but for a wind of $4.7 \mathrm{~m} \mathrm{~s}^{-1}$ speed from east

the geostrophic currents force it away from this direction. This necessary generates strong counter-balancing topographic restoring forces which result in increased vorticity making the current prone to gyre formation. On the other hand, for transverse winds the initial geostrophic and topographic effects operate both towards a relaxation of the topographic restoring forces and lock the flow into the along-channel direction. The increased varied motion in the former case also leads to significantly larger vertical velocities: up-welling and down-welling maximum velocities in the basin under longitudinal winds are $43-67 \%$ greater than the ones under the action of cross winds. The vertical movement is a source of additional vorticity: current convergence results in down-welling and additional cyclonic vorticity, divergence causes up-welling and adds anti-cyclonic vorticity (Gill, 1982). Under winds blowing across the basin, the vertical movement is much less intense; so, as a result, the general basin circulation does not possess enough vorticity to form a local gyre.

Even though these arguments should provide sufficient reason that the gyre formation is physically realistic we performed a number of computations giving support for the claims. Doubling the number of grid points (corresponding to $\Delta x=102.5 \mathrm{~m}, \Delta y=80 \mathrm{~m}$ ) did not alter our results, ensuring results and interpretations are, therefore, trustworthy.

Of special interest is to see how the length and orientation of the peninsula will affect the current patterns in the basin. The gyres are clear indicators of this current structure, which could well show this influence. However, we have seen that gyres are formed in the basin without peninsula only for winds acting in the long direction. For winds from the west one cyclonic 


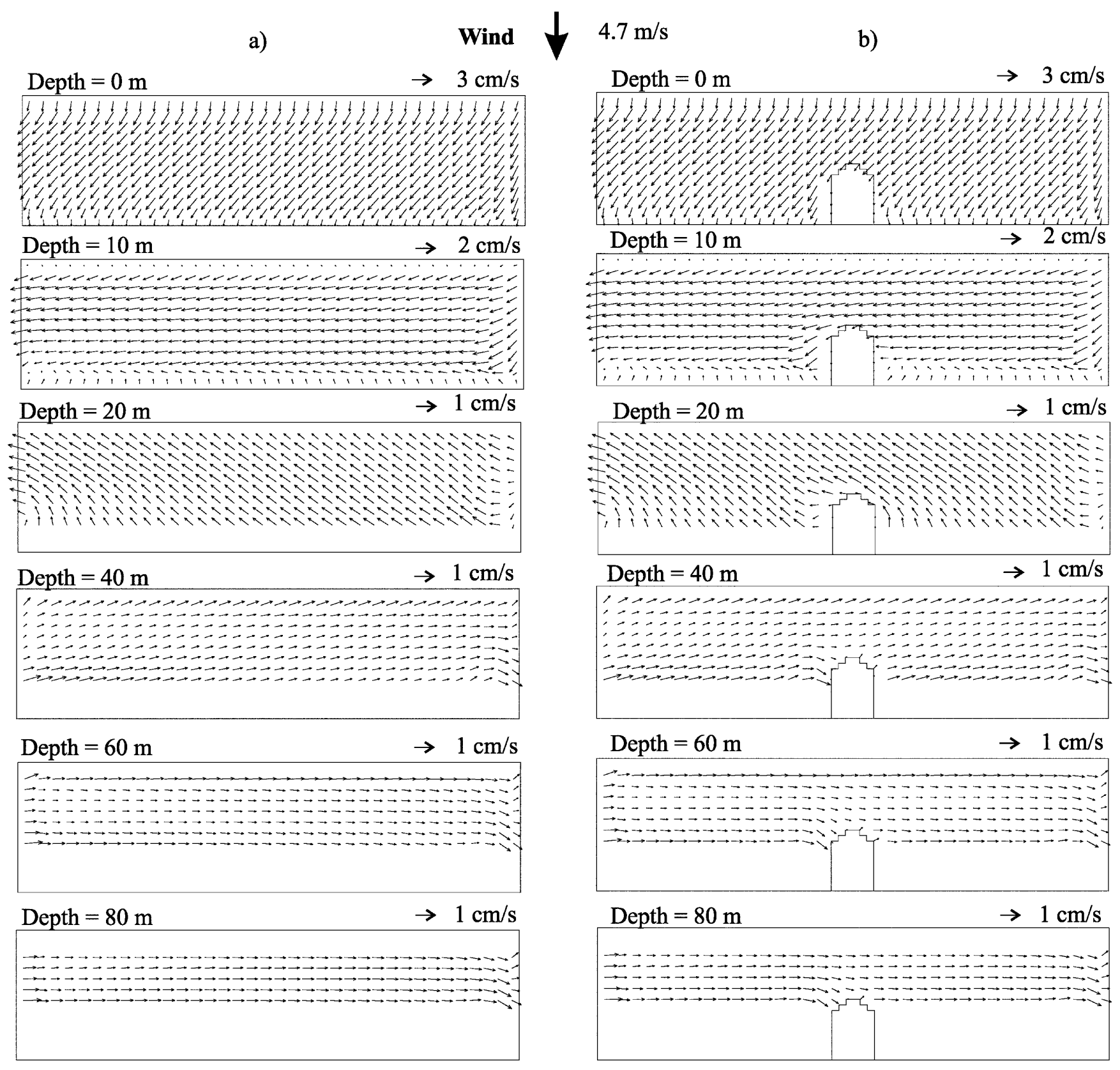

Fig. 6a, b. Same as Fig. 4, but for a wind of $4.7 \mathrm{~m} \mathrm{~s}^{-1}$ speed from north

gyre is formed at the eastern end of the basin, and it extends over the entire depth of the basin. For winds from the east such an anti-cyclonic gyre of less strength is also formed, but it seems to extend only down to $20 \mathrm{~m}$; at greater depths another basin-filling anti-cyclonic circulating flow is formed. For winds from north or south no circulating currents appear in the elongated basin; except for local effects the global current pattern is practically undisturbed by the presence of the peninsula. So, Fig. 8 shows schematically how the peninsula transforms the global current structure for west and east winds. For different lengths of the peninsula sketches of the gyre structure are compiled, which are based on a large number of horizontal current pattern slices made through the depth of the basin.
As is evident from Fig. 8, for a sufficiently short peninsula the obstruction only marginally changes the current pattern very close to the peninsula. No additional gyre is formed, and the currents are slightly diverted by the peninsula. For all lengths of the peninsula greater than a critical length (here it is approximately $0.56 \mathrm{~km}$ ) the current patterns for winds from the west and the east will change, and additional gyres will be formed near the peninsula. So, the presence of the peninsula of more than critical length amounts essentially to cutting the basin into two subbasins each of which is qualitatively subjected to the same current structure as the unobstructed basin. Most conspicuously, a gyre is formed to the left of the peninsula at its end; its location is the eastern end of the 


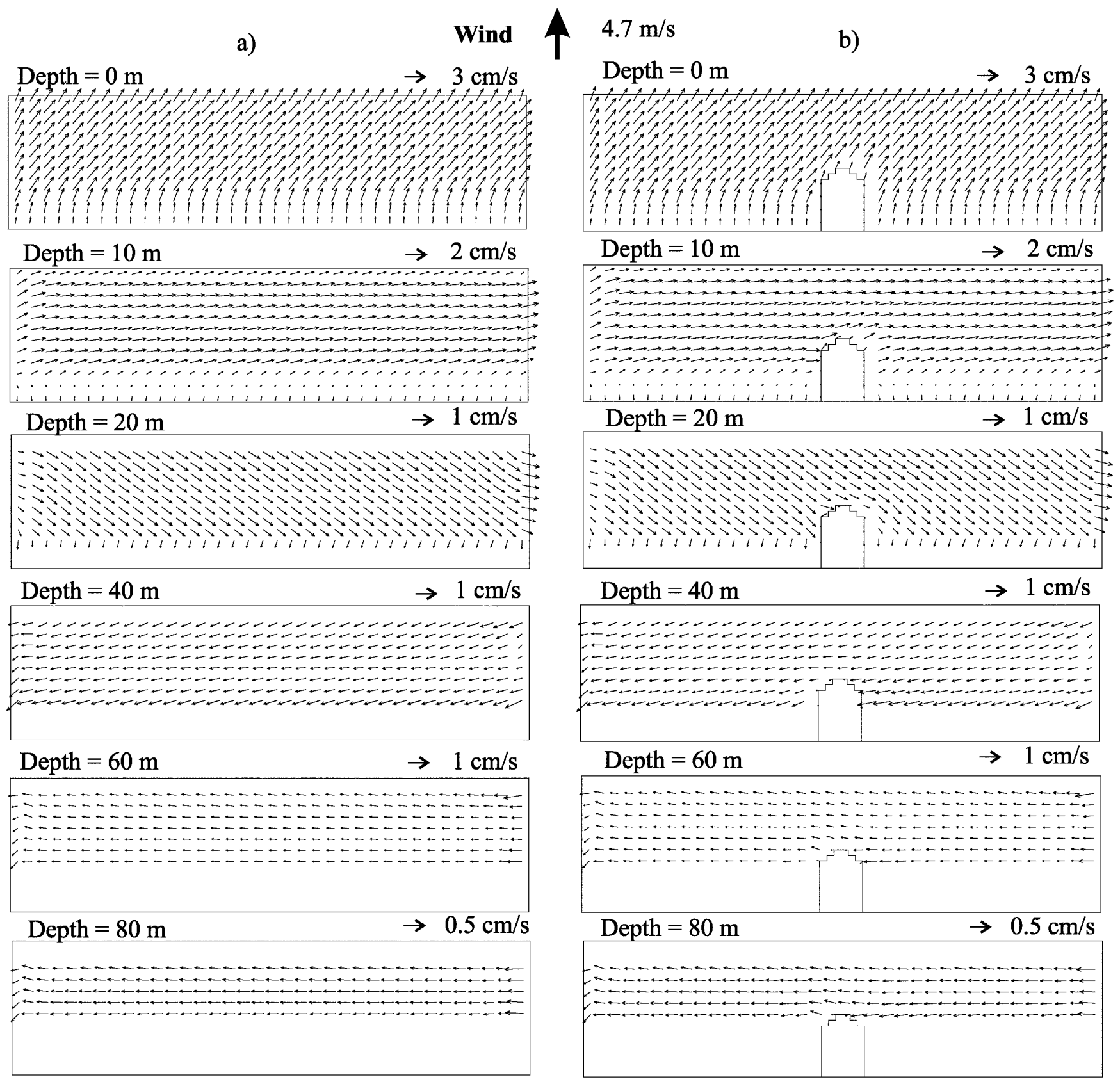

Fig. 7a, b. Same as Fig. 4, but for a wind of $4.7 \mathrm{~m} \mathrm{~s}^{-1}$ speed from south

western basin, and structurally the same current pattern is repeated in the eastern sub-basin. As before, the absolute values of the velocities are larger for easterly winds than for westerly winds, and the coastal jets are also similar as before.

It appears better not to use as a critical parameter the length of the peninsula, but instead to use the relative cross-sectional area, blocked by it, or its complement, which for brevity will be called the relative "passing area" (see Table 1, Fig. 2). In these terms, peninsulas blocking more than $14 \%$ of the basin cross-sectional area (so, the relative passing area is $86 \%$ ) for both east and west winds will hydrodynamically separate the two subbasins each having its own circulation with limited interaction.

\subsection{Water exchange between two sub-basins}

The peninsula in the middle of the long southern shore defines a dividing cross section separating the basin into two sub-basins of equal size. The water exchange through it is a measure e. g. of the renewal of the water in one sub-basin if all inflow and outflow occurs in the other sub-basin. It is interesting to quantify this flux, which is the cross-sectional integral of the $x$-velocity component over the region where it is positive, as a function of the size of the peninsula. Clearly, because under steady conditions with balancing in- and outletbasin fluxes, the total flux through this cross section must be zero, i.e. the fluxes to and fro through the passing cross section are equal. 


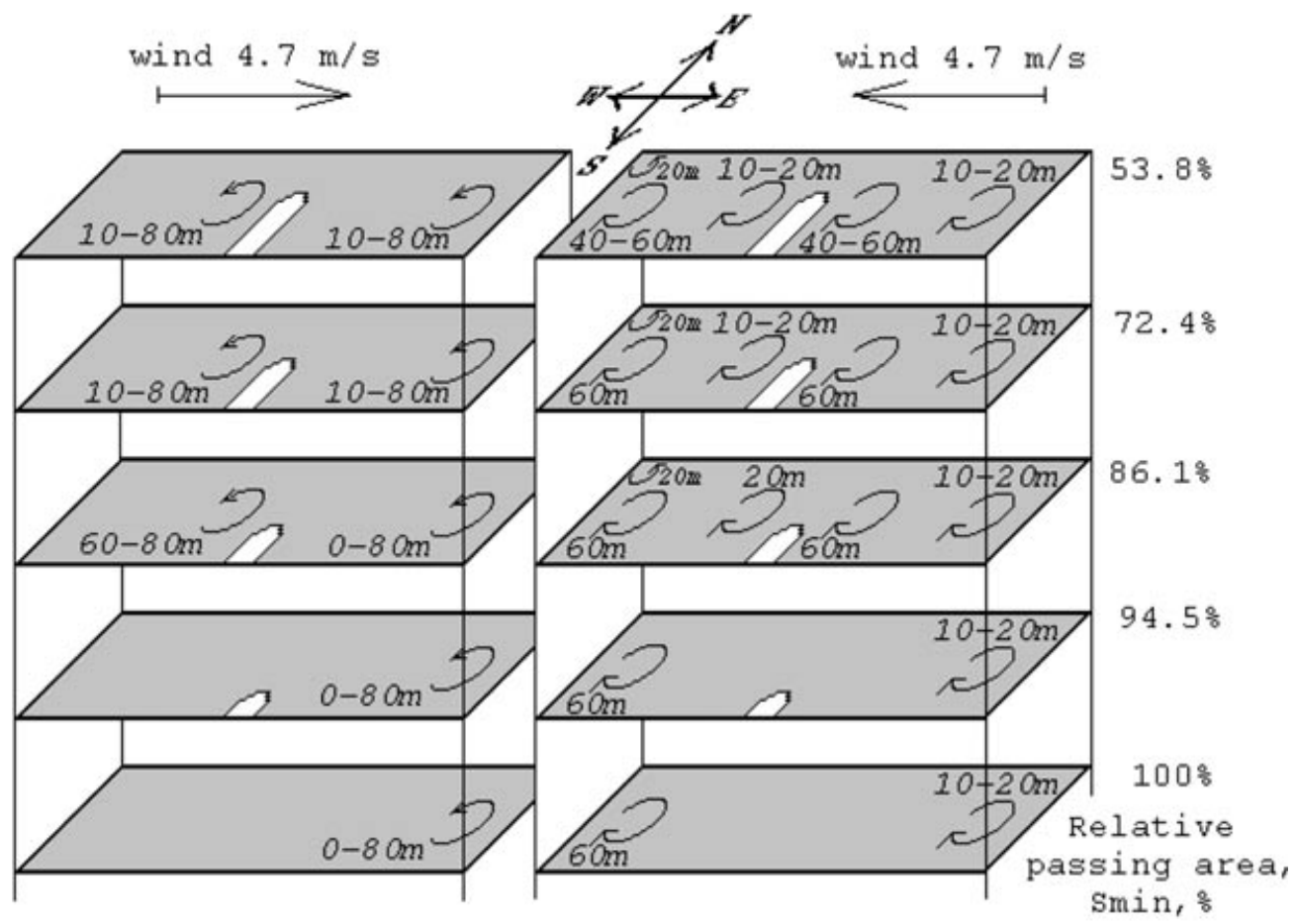

Fig. 8a, b. Sketches of the structure of the steady current in the rectangular basin of Fig. 2 with various peninsulas of different sizes. Indicated are for a west winds and $\mathbf{b}$ east winds the gyre structures as occurring depth ranges as shown (e.g. 10-80 m means that the gyre as sketched is seen in the velocity plots at the depths 10 to $80 \mathrm{~m}$ ). The right column shows the percentage of the passing area relative to the original cross-sectional area without obstruction
Later we will analyse the exact distribution of the velocity components in the three spatial directions in this cross section; here we study the absolute values of the positive (and negative) longitudinal velocity component. Figure 9 and Table 2 summarise the results of the computations. They tabulate and display the flux of the water from one sub-basin to the other through the passing cross section as a function of the relative passing cross-sectional area; this is done for the four wind scenarios shown as insets. The relative error (difference between positive and negative fluxes for all wind directions) in the computed fluxes is about $1.5 \%$. This corroborates the high accuracy claimed for our three dimensional computations. Note also that the four curves suggest different behaviour of the exchange processes for winds in the long and short directions of the basin. Table 2, Fig. 9 and overall current patterns (not shown here) allow us to draw the following inferences.

a. Winds from the north and the south The influence of the peninsula on the exchange water flow between the sub-basins under winds from north and south is always smaller than with winds from west and east. The flux through the passing cross section increases approximately linearly with an increase of the passing crosssectional area. Thus, if $q=Q / Q_{0}$ is the relative flux and $s=S / S_{0}$ the relative passing cross-sectional area, (with

Table 1. Typical parameters of the peninsula with axis perpendicular to the shore line. The "relative length" (in \%) is the ratio of the peninsula length to the total basin width; the "relative passing
$Q_{0}$ the flux and $S_{0}$ the cross-sectional area without the peninsula) then

$q=\alpha s, \quad$ where $\left\{\begin{array}{l}\alpha=0.99 \pm 3 \% \text { for southerly wind } \\ \alpha=0.92 \pm 6 \% \text { for northerly wind }\end{array}\right.$

is an optimal fit; $\alpha$ may be interpreted as a mean nondimensional velocity by which the water exchange occurs through the passing cross section. There follows: the water exchange regime under north and south winds experiences no qualitative changes with growing length of the peninsula. The total flux through the passing crosssectional area from one sub-basin to the other grows nearly linearly with the passing cross-sectional area; i. e. the average velocity of the current perpendicular to the minimum cross-sectional area is nearly the same (with a variation of less than 6\%) for all sizes of the peninsula.

There are some limited differences between the water exchange processes for winds from the north and south, respectively. For instance, the absolute value of the water exchange through the central cross section for the rectangle without the peninsula is larger by $5.5 \%$ for a wind from north than from south (Table 2), and the maximum absolute velocity at the surface and at $40 \mathrm{~m}$ depth is larger by $6 \%$ and $22 \%$, respectively (not shown here). This qualitative behaviour persists only for small peninsulas, whereas the water exchange for south wind

area" is the ratio of the area of the cross section from the tip of the peninsula to the opposite shore to the cross-sectional area if the peninsula would not be there

\begin{tabular}{lcccccccccc}
\hline Length of peninsula (m) & 320 & 800 & 1280 & 1440 & 1760 & 1920 & 2180 & 2240 & 2720 & 3200 \\
Relative length of peninsula (\%) & 8.3 & 20.8 & 33.3 & 37.5 & 45.8 & 50 & 54.2 & 58.3 & 70.8 & 83.3 \\
Relative passing area (\%) & 98.62 & 94.46 & 86.07 & 82.12 & 72.42 & 66.7 & 60.5 & 53.83 & 32.41 & 12.40 \\
\hline
\end{tabular}


a) Absolute flux through minimum basin cross section

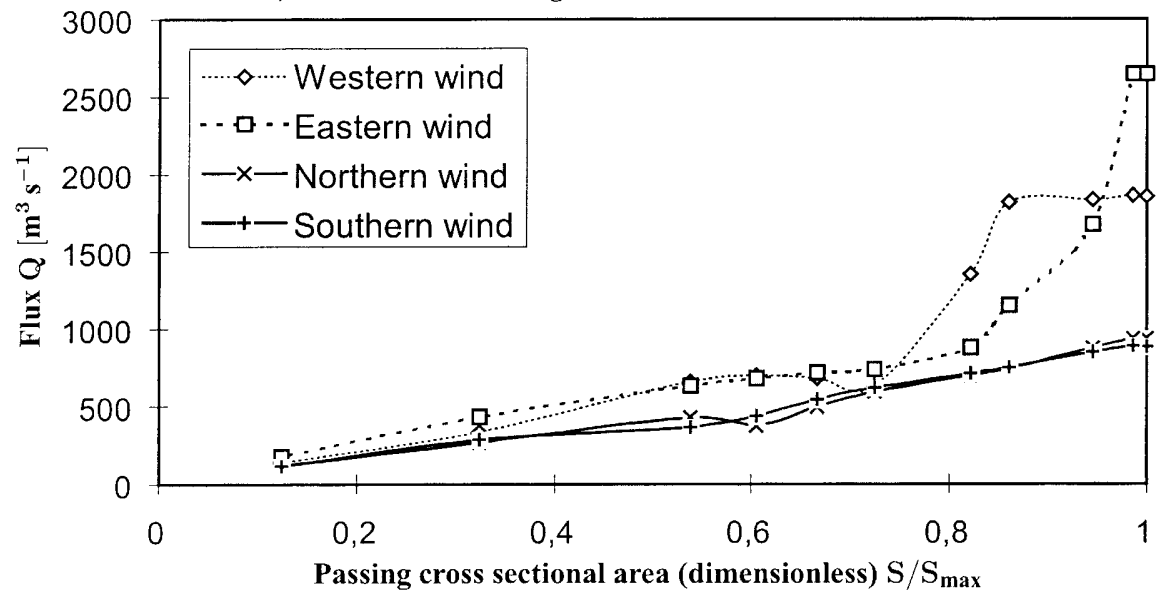

b) Relative flux through minimum basin cross section

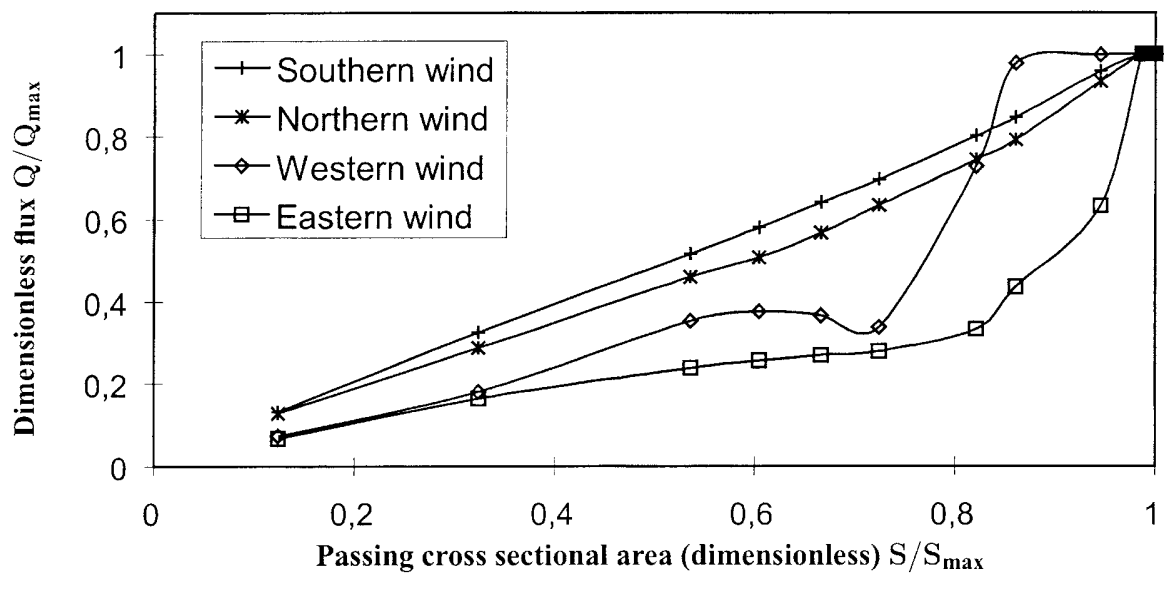

Fig. 9 a Absolute flux (in the positive and negative direction, respectively) through the minimum basin cross section plotted against the relative cross-sectional area $[=($ cross section)/(cross section without peninsula) $\left.=S / S_{\max }\right]$ for four different winds from west, east, north, south. Symbols are computed and taken from Table 2, lines are interpolated. b Relative flux [ $=($ absolute flux $) /\left(\right.$ flux without peninsula) $\left.=Q / Q_{\max }\right]$ through the minimum cross section as in a

Table 2. Absolute flux of water $Q$ (in $\mathrm{m}^{3} \mathrm{~s}^{-1}$ ) through the passing cross section for four different wind scenarios and uniform wind speed of $4.7 \mathrm{~m} \mathrm{~s}^{-1}$ (a medium storm) from west, east, north and

\begin{tabular}{llllllllllll}
\hline Relative passing area & 1 & 0.99 & 0.94 & 0.86 & 0.82 & 0.72 & 0.67 & 0.61 & 0.54 & 0.32 & 0.13 \\
\hline Absolute water flux, $\left[\mathrm{m}^{3} \mathrm{~s}^{-1}\right]$ & & & & & & & & & & & \\
$\quad$ Western wind & 1859 & 1865 & 1837 & 1818 & 1356 & 630 & 683 & 701 & 659 & 336 & 137 \\
$\quad$ Eastern wind & 2648 & 2646 & 1675 & 1155 & 881 & 739 & 716 & 681 & 633 & 434 & 179 \\
$\quad$ Northern wind & 936 & 938 & 874 & 742 & 698 & 593 & 496 & 377 & 431 & 270 & 121 \\
$\quad$ Southern wind & 887 & 890 & 850 & 751 & 712 & 618 & 545 & 436 & 368 & 288 & 116 \\
Relative water flux $\left[\mathrm{m}^{3} \mathrm{~s}^{-1}\right]$ & & & & & & & & & & \\
$\quad$ Western wind & 1 & 1 & 0.998 & 0.978 & 0.729 & 0.339 & 0.367 & 0.377 & 0.354 & 0.181 & 0.074 \\
$\quad$ Eastern wind & 1 & 1 & 0.633 & 0.436 & 0.333 & 0.279 & 0.270 & 0.257 & 0.239 & 0.164 & 0.068 \\
$\quad$ Northern wind & 1 & 1 & 0.934 & 0.793 & 0.745 & 0.634 & 0.567 & 0.508 & 0.460 & 0.288 & 0.129 \\
$\quad$ Southern wind & 1 & 1 & 0.958 & 0.847 & 0.803 & 0.697 & 0.641 & 0.581 & 0.517 & 0.325 & 0.131 \\
\hline
\end{tabular}

becomes larger by $2 \sim 13 \%$ for medium peninsula lengths (Table 2). Furthermore, the current structure in plan view and the structure of up-welling and downwelling regions becomes more complex when the size of the peninsula grows for northerly winds than it does for winds from the south. The larger viscous effects and the guidance of the current by the peninsula in the off-shore direction by the southern winds is the likely cause for this. Complexity of the currents generally limits the size of the current speeds and thus also reduces the absolute value of the water exchange for medium peninsula south and relative flux $Q:=Q / Q_{\max }$ (where $Q_{\max }=Q$ without peninsula) for the same wind scenarios lengths. It follows also that the relative exchange flow is larger (by $8 \%$ on average) for winds from south than for winds from north. This suggests the other bold rule: the more complex the current structure in a sub-basin is, the smaller will be the water exchange between the sub-basins.

b. Winds from the east and the west The results shown could not contrast more with the behaviour of exchange flow under winds in the long direction of the basin, see Fig. 9 and Table 2. Here, no linear dependence of the absolute or relative flux through the passing cross- 
sectional area as a function of the relative area can be discerned. The curves in Fig. 9 for east and west winds are also qualitatively different from one another and need detailed explanation.

The absolute value of the water exchange through the central cross section without peninsula is 1.42 times greater with winds from the east than for those from the west (Table 2). The strong coastal jet near the southern shore, which is more intense and wider for winds from the east (see Figs. 4 and 5), gives rise to larger absolute flux values for east winds than for west winds (Fig. 9a). For western winds the gyre in the empty basin is more intense and more localised than for easterly winds, it embraces the entire depth and has velocities three to five times larger than in the remainder of the basin. Contrary to this, the vortex structures for easterly winds occur only at 20-30 and $60 \mathrm{~m}$ depths, possess smaller velocities and embrace most of the basin. This localisation of the momentum in the local gyre for winds from west reduces the mechanical movement in the remainder of the basin (since the total kinetic energy stored in the basin must be similar in the two cases) and therefore reduces the water exchange. This is then a typical behaviour, also observed in other wind and peninsula scenarios: whenever a gyre is formed the water exchange between the two basins decreases significantly. The reverse is, however, not true: a decrease in water exchange between the basins is not necessarily the evidence of the formation of a gyre, but may be due to some rearrangement of the currents.

In this light the curves in Fig. 9 ought to be interpreted. For instance, for easterly winds the flux through the passing cross section decreases by $37 \%$ when the passing cross section is reduced from its maximum by $6 \%$ (Fig. 9, Table 2). However scrutiny of the velocity plots (not shown here) reveals that this is not the result of the appearance of a new vortex zone in the basin, as the horizontal current distribution is merely distorted from the form it shows without a peninsula. In particular, the along-shore velocity components in the vicinity of the southern shore are reduced which contributes to the mentioned flux reduction. The reduction of the area of the passing cross section on the next $12 \%$ causes an additional $30 \%$ reduction of the through-flow (Fig. 9, Table 2), but now the current structure is qualitatively changed (Fig. 8). If the length of the peninsula is further increased (Fig. 8) a gyre is formed also in the western sub-basin. This behaviour is the likely reason why the flux curve in Fig. 9 for eastern winds is essentially a monotonic function of the passing cross-sectional area.

For westerly winds the water exchange between the left and right sub-basins remains essentially constant at first as the peninsula grows from zero to a critical size; indeed, the first $12 \%$ reduction of the passing cross section reduces the flux through the minimum cross section only by $2 \%$ (Fig. 9). A further increase of the size of the peninsula, however, results in a sudden drop of the water exchange (at relative passing areas from 0.86 to 0.72 ), and this is obviously due to the inception of the cyclonic vortex immediately to the northwest of the tip of the peninsula. Velocity plots, not shown here, indicate that this gyre arises first in the bottom layers $(60-80 \mathrm{~m})$ but extends later over the entire depth. This is the critical transition, and it leads to a rearrangement of the currents in the sub-basins which are now similar to those of the entire basin without peninsula.

The reason for the local minimum of the flux under western winds (Fig. 9) lies in the dynamics of the cyclonic gyre appearing to the left of the peninsula (Fig. 8). Being "born" actually in the strait, this gyre is squeezed out to the left as the peninsula is increased. At its full strength this gyre extends roughly over two thirds of the basin width. The local minimum of the flux corresponds to a flux reduction by $72 \%$ (Table 2). It occurs when the flow in the minimum cross section is essentially in the $y$-direction. A further narrowing of the strait leads to a continuous supplanting of the gyre to the west and a partial embedding of the minimum cross section in the circulation of the eastern sub-basin. For this situation the border of the two sub-basins is not the minimum cross section but a curved area to the west of it. An even further reduction of the cross section now results in essentially a linear drop of the exchange flow.

We also notice that the critical transition between the two regimes of the water exchange for any wind arises roughly when the width of the connecting channel has reached the size of a gyre inherent for the given system.

\subsection{Current structure in the minimum cross section}

The preceding analysis dealt with a global quantity, the water exchange. It is instructive to see how all three velocity components are distributed over the minimum cross-sectional area and how this distribution changes as the peninsula grows. This transition is a general transformation of the three-dimensional current pattern in the entire basin. The limited information provided by the velocity distribution within the minimum cross section gives nevertheless rather detailed information about the three-dimensionality of the current in its vicinity, when the size of the peninsula grows. Figures 10-13 summarise the results for the same four wind scenarios used already. The current structure in the minimum cross section changes in a qualitative way only for winds in the long direction of the basin but remains principally the same for winds across the basin when the size of the peninsula is varied. This is plausible because the peninsula is a bigger obstruction for winds in the long direction than those across the basin.

The $x$-components of the current (left columns of Figs. 10-13) provide detailed information about the distribution of the exchange current. For winds from west and east, as the peninsula becomes larger, these currents experience two types of characteristic transitions. When no peninsula is present, the near-shore flow and the surface flow is with the wind whilst the off-shore core flow is against the wind, as physically expected, see e.g. Csanady (1984). As the peninsula becomes bigger, this flow profile is squeezed to smaller widths but qualitatively preserved (Figs. 10, 11). This behaviour 

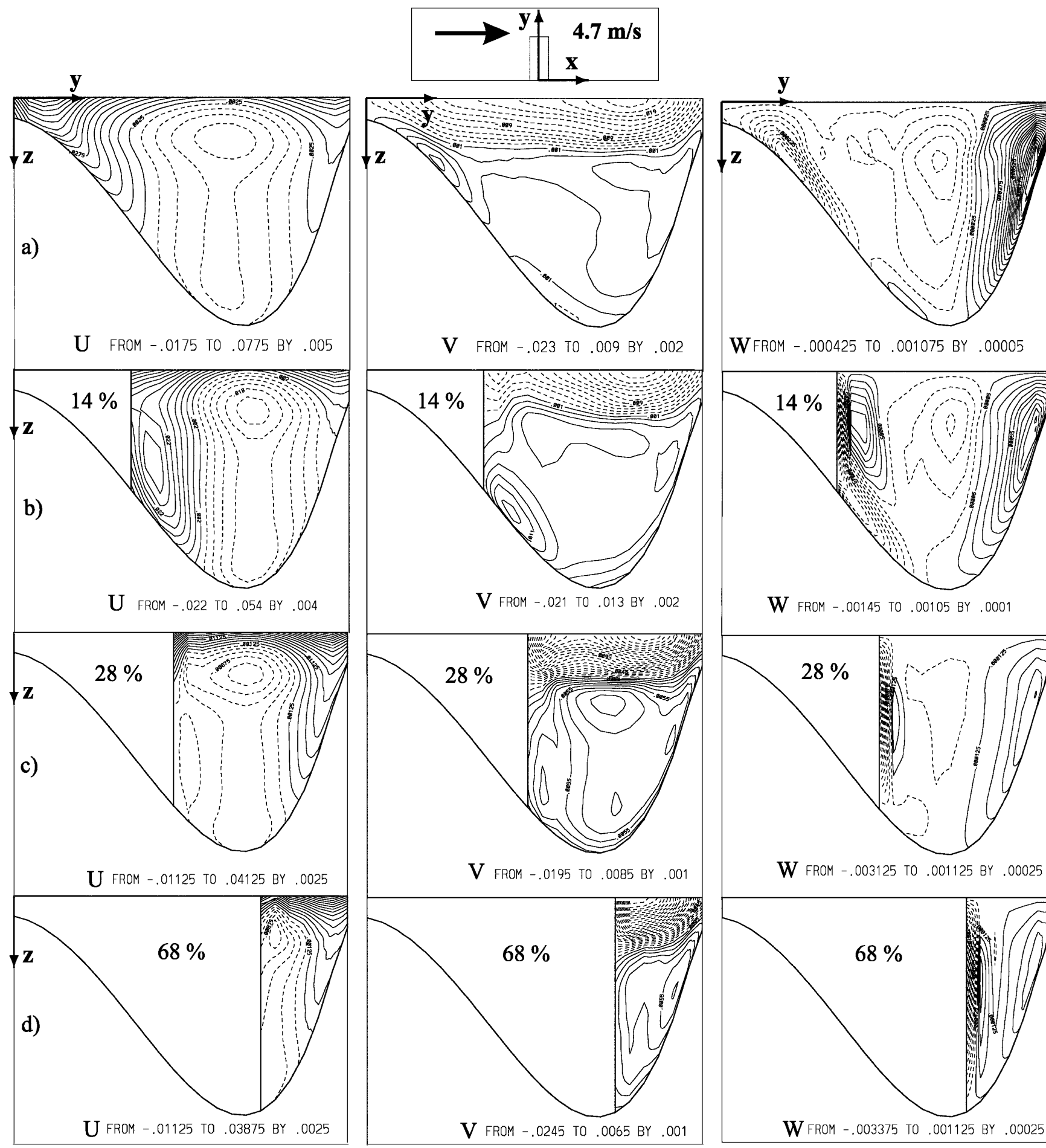

WFROM -.000425 TO .001075 BY .00005
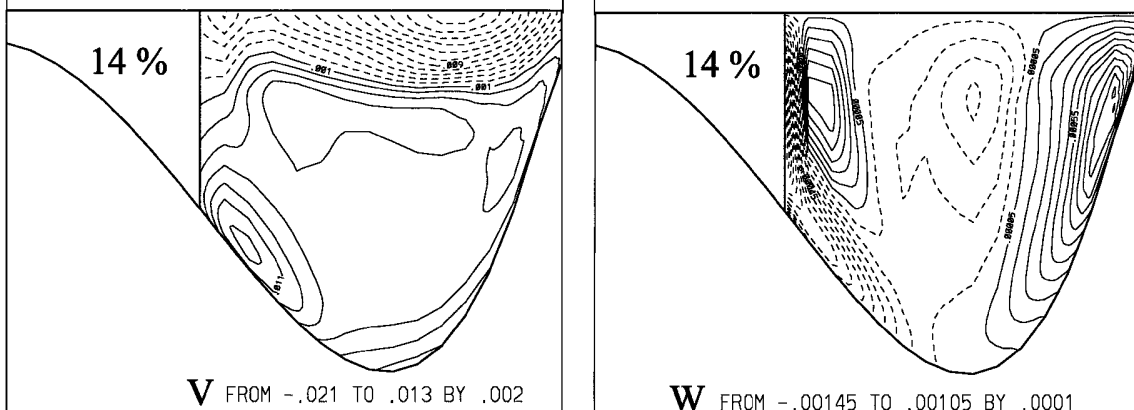

W FROM -.00145 TO .00105 BY .0001
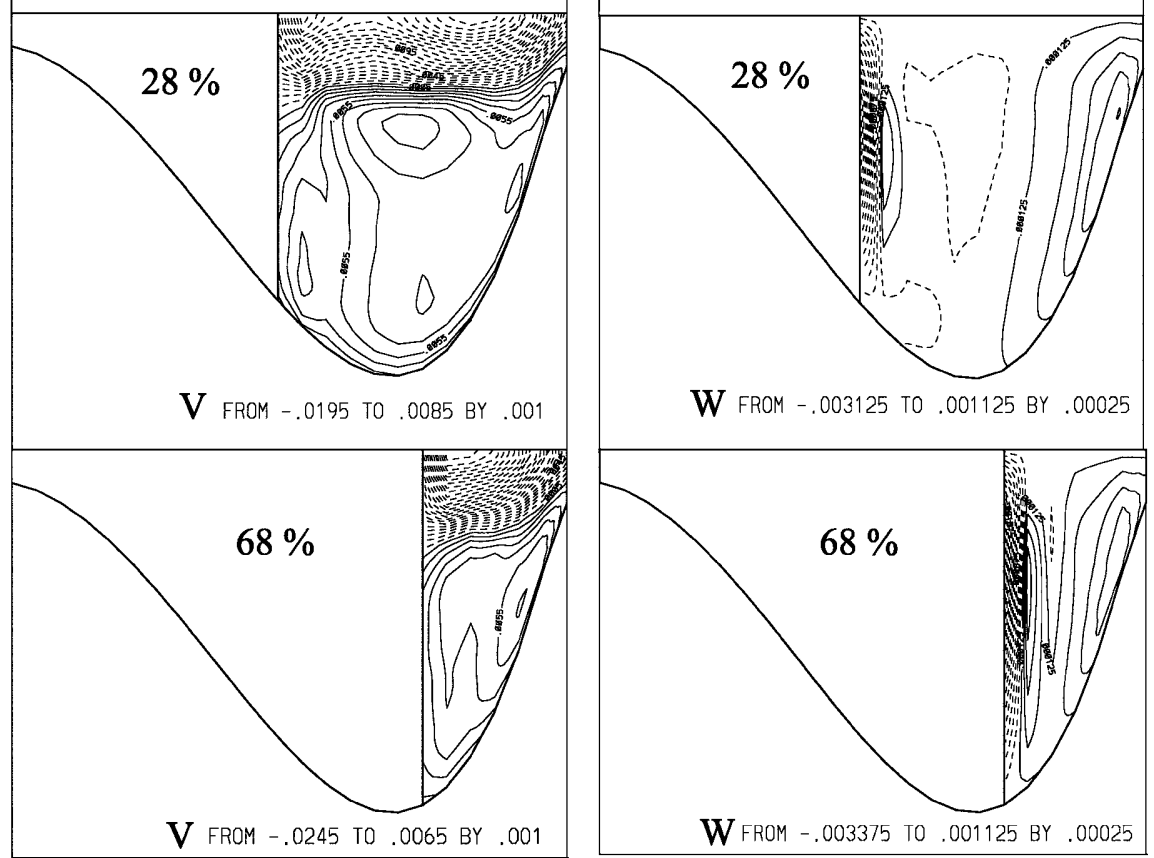

Fig. 10a-d. Velocity distribution in the cross section connecting the midpoint at the southern shore (left side of each graph) with the midpoint of the northern shore (right side of each graph). The white area in each panel shows the area covered by the peninsula and the solid curve shows the bottom topography. The graphs show isotachs for the velocities in the $x$-(left columns), $y$-(middle column) and $z$-directions (right panels), in which the directions of the positive

$u$-, $v$ - and $w$-velocities are defined as eastward, northward and upward velocities, respectively; solid (dashed) lines indicate positive (negative) velocity components and labels are given in $\left(\mathrm{m} \mathrm{s}^{-1}\right)$. The graphs are valid for a wind from west with $4.7 \mathrm{~m} \mathrm{~s}^{-1}$ when steady conditions are reached. The four cases of peninsula length are presented: a no peninsula, b peninsula blocks $14 \%$, c $28 \%$ and d $68 \%$ of the initial cross section

lasts until the passing cross section is reduced to about two thirds of its original size, where the new sub-basin gyre is formed to the west of the peninsula. For even smaller passing cross sections (Figs. 10, 11c, d) the

windward current at the front end of the peninsula disappears. This marks the critical size of the peninsula. With even larger lengths of the peninsula, the throughflow with the wind is primarily along the northern shore 


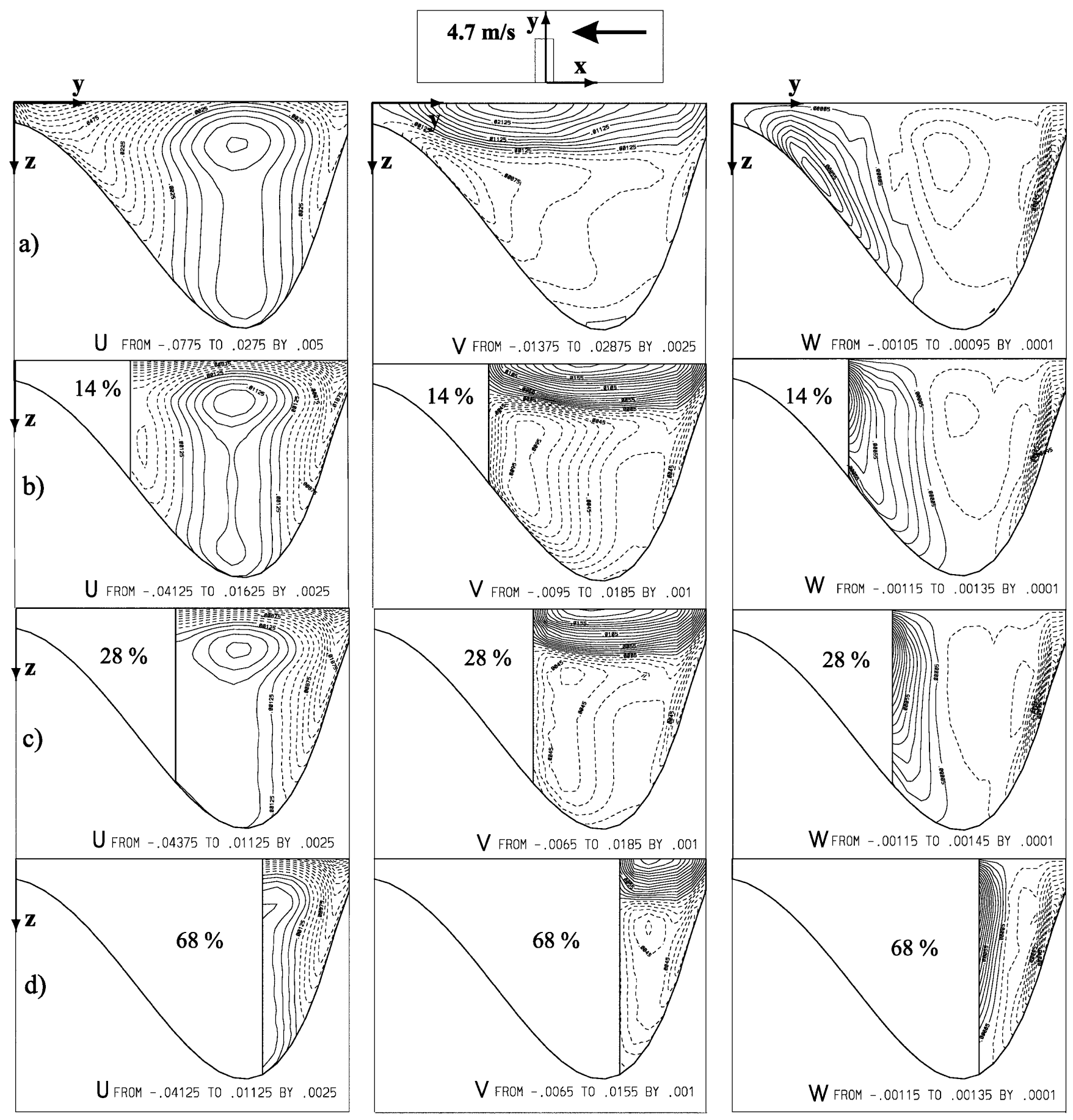

Fig. 11a-d. Same as Fig. 10 but for a wind from east

and at the top whilst the return current maximum is close to the peninsula.

For east and west winds the transverse flow (middle columns of Figs. 10,11) is to the right of the wind in a (thick) surface layer with a return current at depth. This behaviour, which is reminiscent of the Ekman drift, does not change as the peninsula is growing. The vertical velocity components (right columns of Figs. 10, 11) show up-welling to the left of the wind and downwelling at the opposite shore. These are due to the set-up structure of the steady water mass distribution. There is one exception to this rule for west winds due to the appearance of a cyclonic gyre and its motion which also leads to an intermediate up-welling zone close to the peninsula (Fig. 10). This can equally be understood by the fact that the enhanced vorticity of the cyclonic gyre gives rise to localized up-welling.

For winds from north and south (Figs. 12, 13) the surface-near flow in the $x$-direction is to the right of the wind with the return flow at larger depths (Ekman drift!). Exception to this is the northern shore, where the return flow reaches the surface and the southern most 

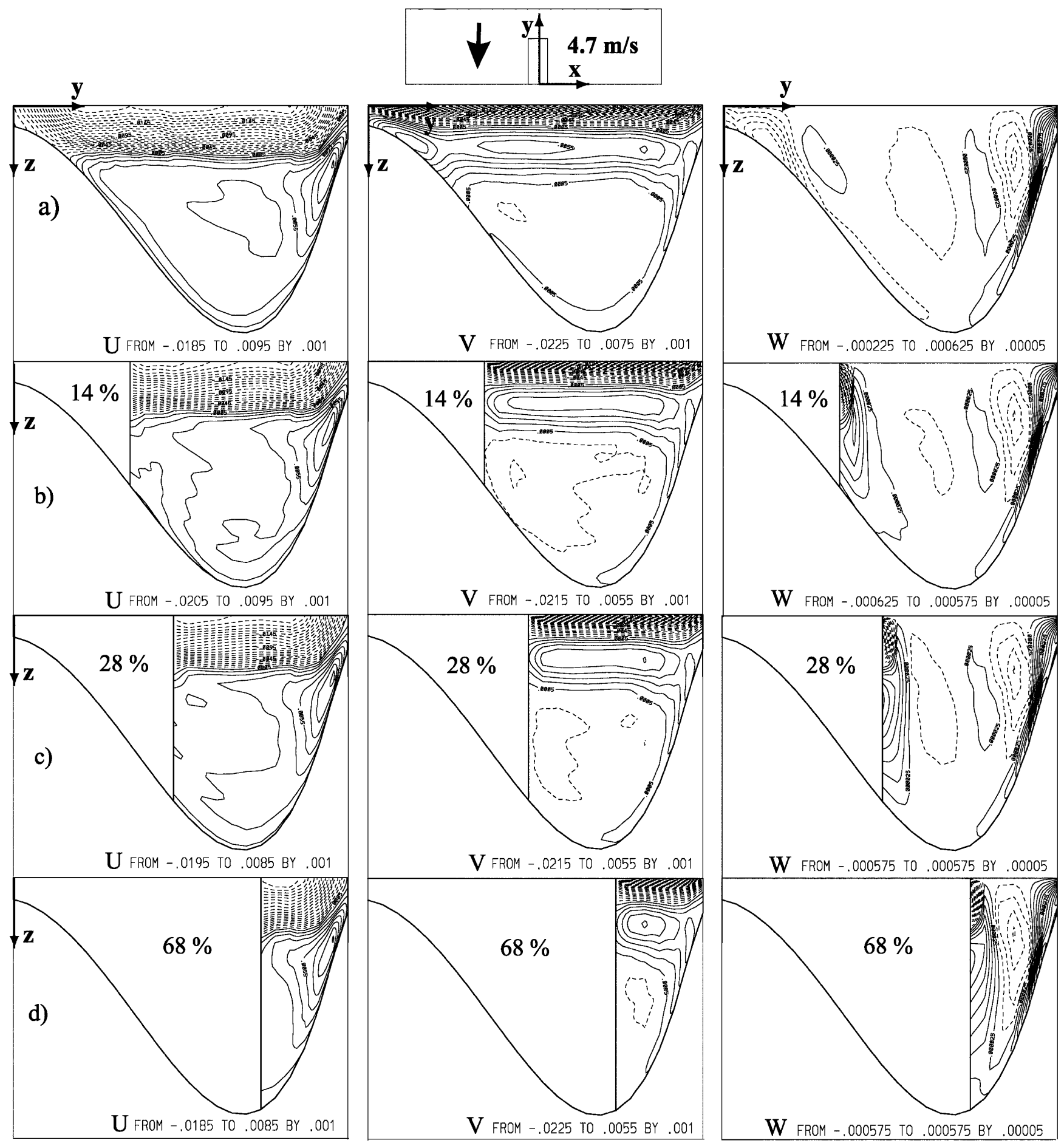

W FROM -.000575 TO $.000575 \mathrm{BY} .00005$

Fig. 12a-d. Same as Fig. 10 but for a wind from north

surface region for no and very small peninsula. The across-basin velocity is with the wind in the top layer and against the wind in the bottom-near layer. The structure of the vertical velocity distribution is very complex. There are several up-welling and down-welling regions, in opposite directions for winds from north and south, but the basic division in up- and down-welling zones does not change as the peninsula becomes bigger. We have not been able to link this behaviour to a particular physical behaviour, but the stability of the structure is indication that the behaviour is not a numerical artifact.

\section{Special features}

In this section we collect a number of findings that point at special properties which are not at the centre of the 

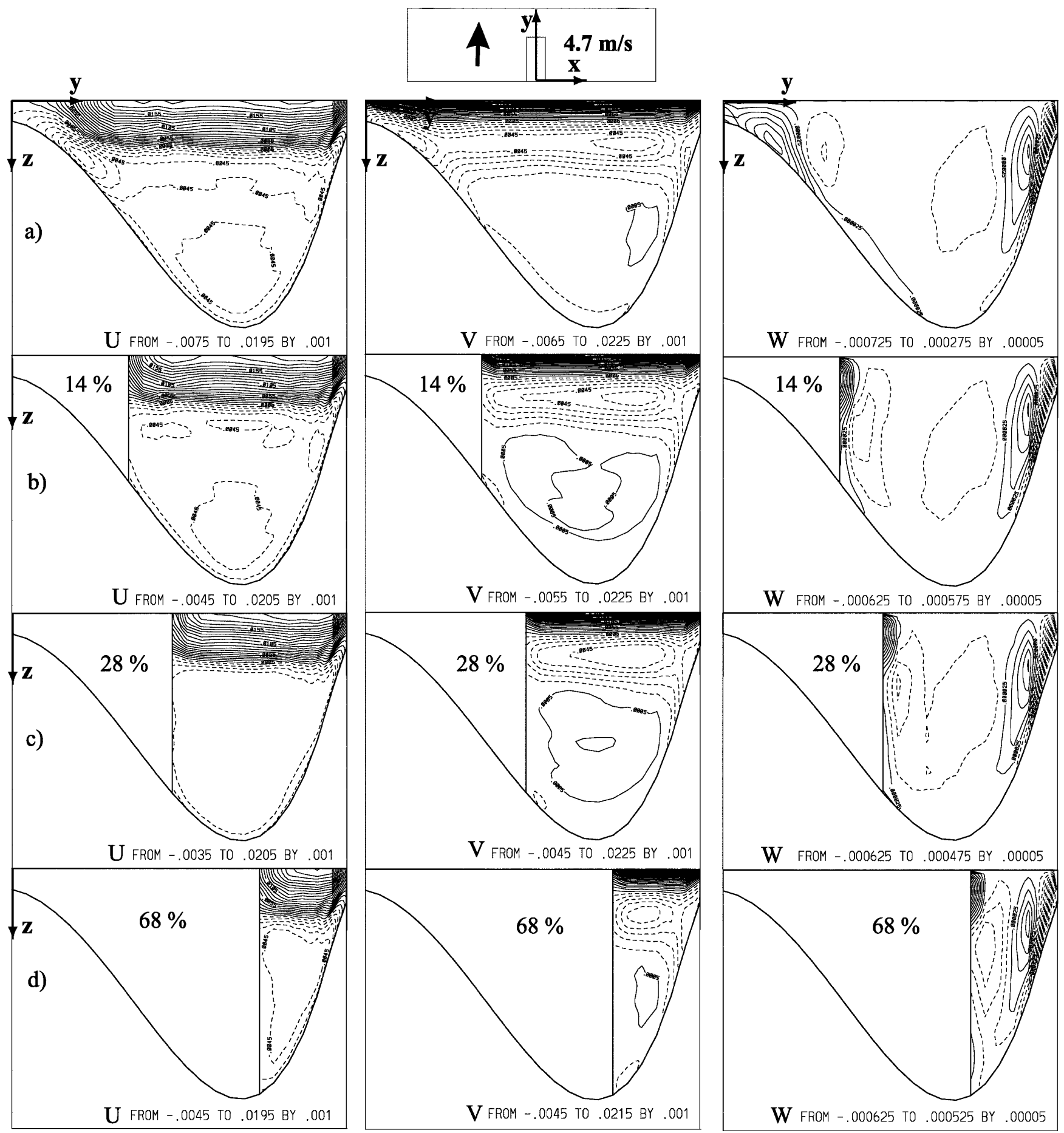

Fig. 13a-d. Same as Fig. 10, but for a wind from south

present study but are nevertheless sufficiently important to warrant being mentioned.

\subsection{Three-dimensional versus two-dimensional modelling}

Barotropic processes in lakes and ocean basins are often studied by using vertically integrated hydrodynamic models. They are considered practical, use small computer memory and are economical in calculations.
However, are they also accurate when being compared with results from three-dimensional model computations? A criterion by which this can be estimated evaluates the ratio of two times

$t_{\text {dif }}=H_{0}^{2} / v_{v}, \quad t_{\text {adv }}=L_{0} / U_{0}$,

in which $H_{0}, L_{0}, U_{0}$ and $v_{v}$ are a typical depth, horizontal length and velocity scales and vertical kinematic viscosity; $t_{\text {dif }}$ is the time it takes a signal to diffuse a vertical distance $H_{0} ; t_{\text {adv }}$ on the other hand, is the time it takes a 
particle to be horizontally advected a distance $L_{0}$ by the speed $U_{0}$. The second time must be much larger than the first for a two-dimensional model to be meaningful; for otherwise a vertical turbulent mixing process has not been completed before a non-negligible horizontal transport has taken place, obvious conditions for the three dimensionality of the processes involved. It is readily seen that the necessary inequality for two dimensionality of the flow is

$H_{0}^{2} U_{0} v_{v}^{-1} L_{0}^{-1} \ll 1$,

despite the fact that it is nearly never satisfied except for extremely shallow basins such as e.g. Neusiedler See (Austria/Hungary). The question whether two-dimensional computations are meaningful, can only be posed with regard to some global variables such as e. g. the water exchange between sub-basins.

This was done in the following way: consider the minimum cross section with distribution of longitudinal velocity components as displayed in Figs. 10-13 in the left panels. For any position $y$ in these panels one may separately sum over all positive and all negative values of the longitudinal velocity. This yields $Q^{+}(y)$ and $Q^{-}(y)$, the specific positive and negative volume fluxes per unit width. The steady state functions $Q^{+}(y)$ and $Q^{-}(y)$ are plotted in Fig. 14 for several wind scenarios and peninsulas. Adding $Q^{+}(y)$ and $Q^{-}(y)$ for a fixed value of $y$ yields the net volume flux $Q_{\text {sum }}(y)=Q^{-}(y)+Q^{+}(y)$ per unit width at $y$, and obviously the sum

$$
\int Q_{\text {sum }}(y) \mathrm{d} y=0
$$

must vanish in steady state. These curves are also shown in the panels of Fig. 14. Imagine now that computations are performed with a two-dimensional, vertically integrated model. Such a model will only be able to generate the variable $Q_{\text {sum }}(y)$ but not $Q^{+}(y)$ and $Q^{-}(y)$. It follows that the two dimensional, vertically integrated model will be able to reproduce the exchange transport adequately, only if the curves $Q_{\text {sum }}$ nearly coincide in the respective domains with $Q^{+}(y)$ and $Q^{-}(y)$, respectively. This happens, if $Q^{+}(y)$ nearly vanishes where the absolute value of $Q^{-}(y)$ is large and vice versa.

Conditions of sufficient agreement in the sense mentioned are obtained for winds from west and east and a short peninsula (Fig. 14a, c). Similar satisfactory agreement is obtained everywhere except in the vicinity of the end of the peninsula for east and west winds and for peninsulas which nearly reach the middle of the basin (Fig. 14b, d). Complete failure would be obtained for winds from north and south and all lengths of the peninsula (Fig. 14e, f). In these cases three-dimensional computation is indispensable.

\subsection{Transformation of up-welling and down-welling zones}

Regions of up- and down-welling identify the zones with intense vertical advection. Under the four wind scenarios and the unobstructed rectangle with variable bottom, the isolines of the vertical velocity essentially follow the isobaths, and up- and down-welling zones occur as detailed in Table 3.

This global pattern remains essentially unchanged when a peninsula is present. What alters is a local influence due to the presence of the peninsula, but its size does not influence its qualitative behaviour. Figure 15 displays isolines of the vertical velocity component at the $10 \mathrm{~m}$ depth level for a rectangle with one particular peninsula and wind scenarios as indicated in the insets. The up- and down-welling structure distant from the peninsula is as described in Table 3, but the shore zones around the peninsula experience strong up- and downwelling (Fig. 15). For west winds, there are three zones of up-welling, one on the downstream side of the peninsula in the upper 15-20 m, one to the west of its front (down to $50 \mathrm{~m}$ depth), and a third (penetrating down to the bottom, caused by the gyre) to the west of the latter. For winds from the east strong up-welling (down to the bottom) arises at the western and northern shores of the peninsula and down-welling (penetrating to the bottom and further along the bottom slope) at the eastern shore of it (Fig. 15b). Both events are highly restricted to the vicinity of the peninsula with a larger spatial extent of the up-welling zone in contrast to the down-welling zone regardless of the wind direction. Down-welling zones are restricted to narrow strips along the shore; for both winds they are accompanied by intense up-welling zones offshore of the down-welling zones. These processes have their positive bearing in the water renewal of the near-shore zone of the peninsula.

For north winds in Fig. 15c (south winds in Fig. 15d) the pattern remains relatively simple: up-welling arises essentially at the western (eastern) shores of the peninsula and down-welling at the east (west) of it. These events are obviously caused by the Coriolis force. These zones of up- and down-welling are more highly restricted to the immediate vicinity of the peninsula than for winds from the west and the east.

The details (e.g. width and strength) of these upwelling and down-welling zones depend on the exact bathymetry around the peninsula. Since the latter is characterised by vertical shores (Fig. 2), which is somewhat unrealistic, we refain from going into greater discussions.

\subsection{From peninsula perpendicular to the shore to inclined peninsula and island}

The preceding analysis dealt with one single configuration: a peninsula with axis perpendicular to the shore line. Here we summarise results obtained for inclined configurations and for islands.

a. Inclined peninsula Computations were performed for configurations as sketched in Fig. 16. Significant differences with the current structure of the peninsula with its axis perpendicular to the coast line were not found. In particular for peninsulas with the same effective length, 

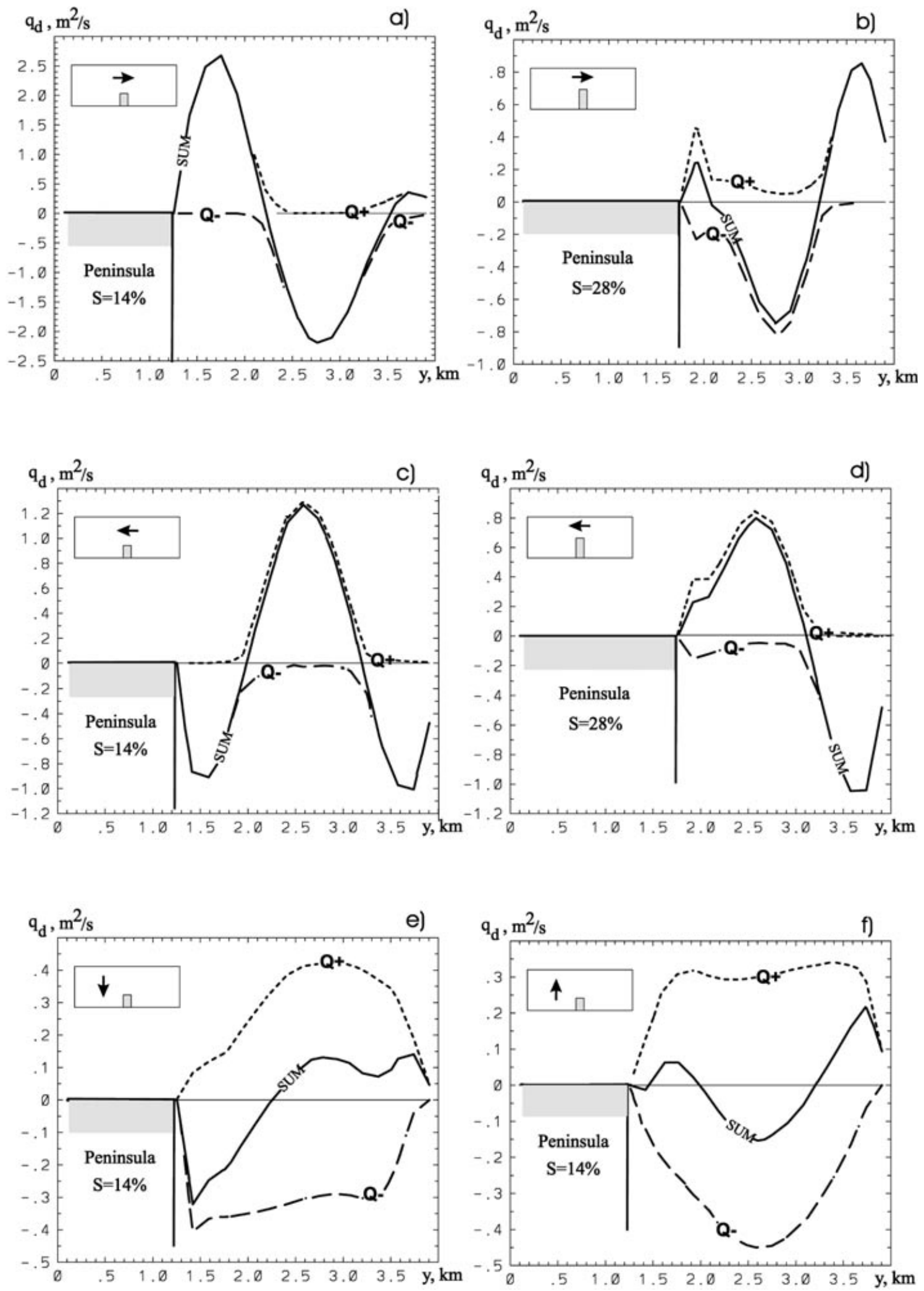

Fig. 14a-f. Vertically integrated positive, $Q^{+}(y)$, and negative, $Q^{-}(y)$, volume flux per unit width in the minimum cross section plotted against distance from southern shore for steady conditions and for

Shown is also the curve $Q_{\text {sum }}(y):=Q^{+}(y)+Q^{-}(y)$. Cases a-d would also be adequately predicted by a two-dimensional model, cases e-f would not do so 
Table 3. Positions of up-welling and down-welling zones for the unobstructed rectangular basin under four wind scenarios

\begin{tabular}{lll}
\hline Wind & Up-welling & Down-welling \\
\hline Western & $\begin{array}{l}\text { Northern and western } \\
\text { shores } \\
\text { Wide southern zone } \\
\text { and narrow band at } \\
\text { eastern shore }\end{array}$ & $\begin{array}{c}\text { Eastern shore and } \\
\text { remainder of the basin } \\
\text { Western shore and } \\
\text { remainder of the basin }\end{array}$ \\
Northern & $\begin{array}{c}\text { Narrow bands at the } \\
\text { northern and } \\
\text { eastern shores } \\
\text { Remainder of the basin } \\
\text { (with intensification } \\
\text { at the western end) }\end{array}$ & $\begin{array}{c}\text { Remainder of the basin } \\
\text { (in particular western } \\
\text { and southern shore) } \\
\text { Narrow band at the } \\
\text { northern and eastern } \\
\text { shores }\end{array}$ \\
\hline
\end{tabular}

i. e. for configurations with the same passing area the water exchange between the two sub-basins turned out to be nearly the same as for the old cases. Differences in the current were nowhere seen to be larger than $1.5 \%$. Variations of the current structure pattern are illustrated in Fig. 16 for east and west winds. The minimum passing cross-sectional area (Fig. 2) is $86 \%$ of the total area. We see, that for easterly winds the vortex structure is the same for all cases. Each sub-basin is subjected to two anti-cyclonic gyres at the indicated depths between $10-20 \mathrm{~m}$ and $60-80 \mathrm{~m}$; in addition, there is a small cyclonic gyre in the NW corner at $20 \mathrm{~m}$ depth. For winds from the west the current pattern is different: for a peninsula or island inclined against the wind the current pattern is disturbed more than when the axis of the obstruction is perpendicular to the shore. This is shown in the extent of the cyclonically rotating gyre to the west of the obstruction, which in the two cases is at the 20
$80 \mathrm{~m}$ and $60-80 \mathrm{~m}$ depth range, respectively. Furthermore, when the peninsula or island is inclined with the wind, there is no gyre formed to the left of the obstruction in this case. Interestingly too, the small cyclonic vortex in the NW corner of the basin is generated only when the obstruction is inclined relative to the shore.

As could be suspected in view of the results of the previous subsection, the intensity and structure of the up-welling and down-welling processes in the immediate neighbourhood of the obstruction experienced larger alterations. As before, with winds from west and east the up-welling zone occurs on the front side of the peninsula or island, and its intensity is about two to three times larger if the direction of the wind and the axis of the peninsula form an acute rather than an obtuse angle.

Some other details are as follows:

1. For westerly winds the down-welling on the front side of the peninsula is about half as large as for peninsulas oriented perpendicular to the shore. This is so for both acute and obtuse angles of orientation of the peninsula.

2. For easterly winds the down-welling at the eastern side of the peninsula is about five times larger than for peninsulas oriented orthogonally to the shore when the orientation angle is obtuse, and twice as large when it is acute.

3. When the wind is from the north, the down-welling bottom velocity to the east of the peninsula is about three times larger for peninsulas with their axis to the east than to the west.

It is evident from these observations that the orientation of the peninsula relative to the wind exercises a major a)

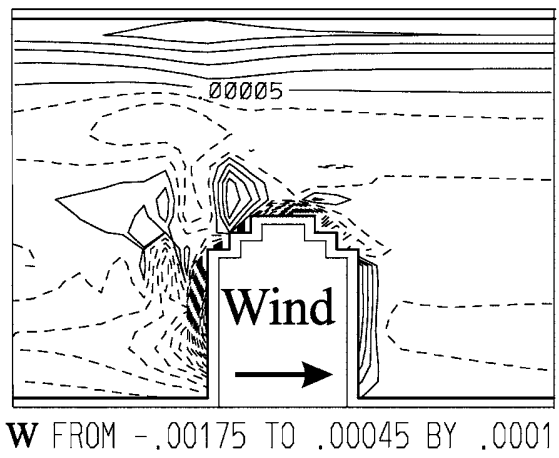

c)

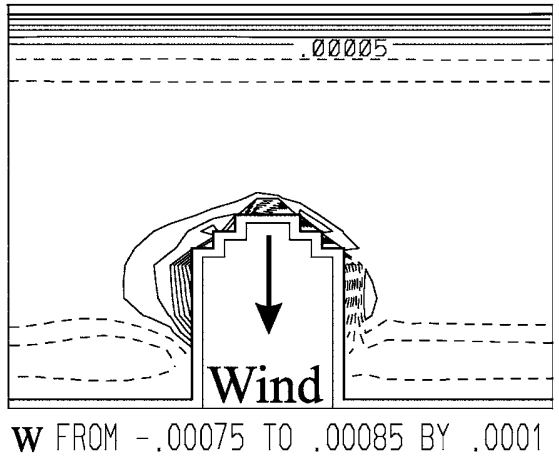

b)

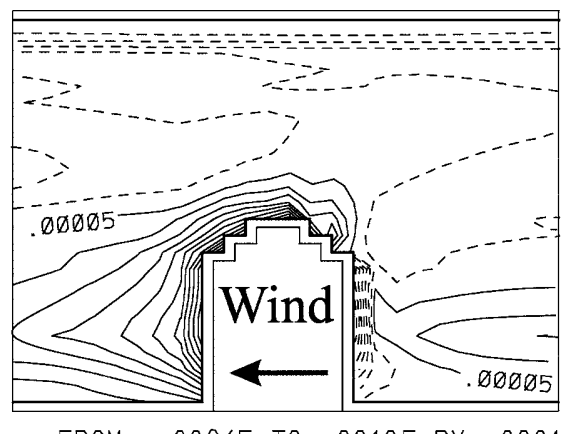

W FROM -.00065 TO .00105 BY .0001

d)

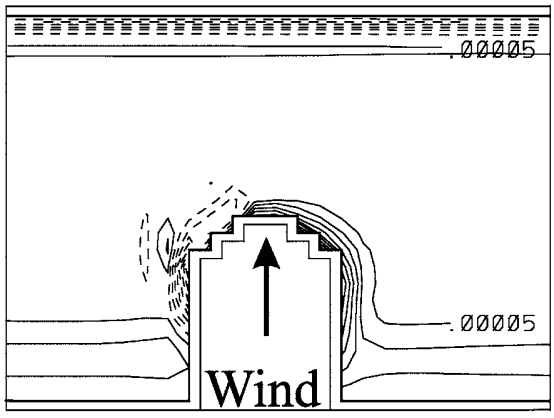

W FROM - .00065 TO .00085 BY .0001
Fig. 15a-d. Regions of up-welling (black) and down-welling (dotted isolines for negative vertical velocities) for winds from a west, $\mathbf{b}$ east, $\mathbf{c}$ north and $\mathbf{d}$ south 

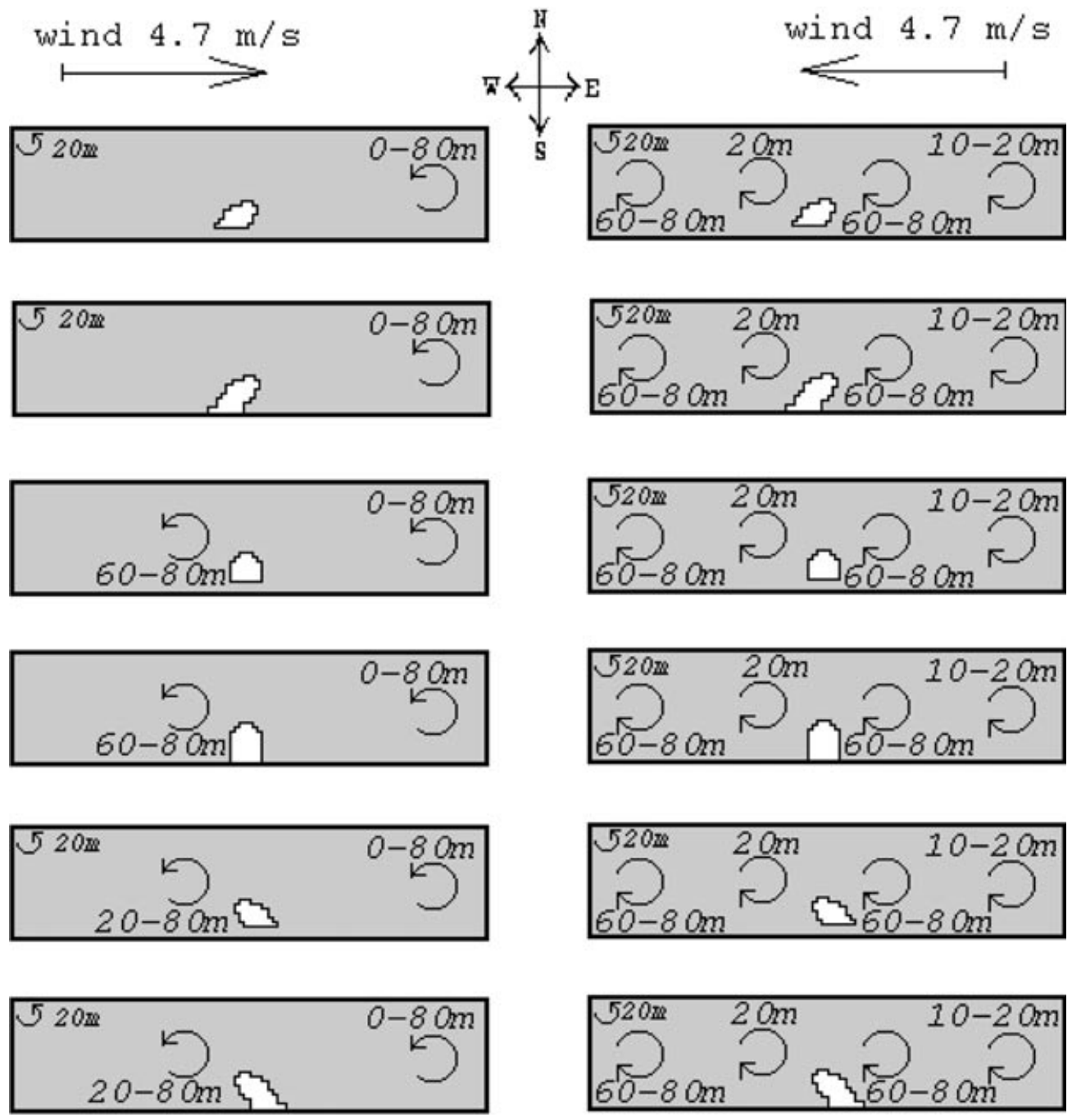

a)

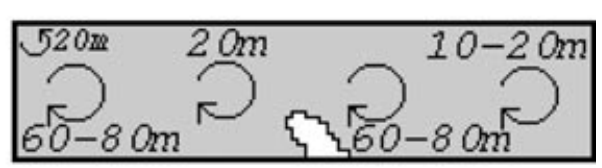

b)
Fig. 16a, b. Sketches of the structure of the steady current in the rectangular basin with various peninsulas and islands of different shapes. Indicated are a for west winds and $\mathbf{b}$ east winds the gyre structures occurring in the depth ranges shown by the numbers. Note the differences between the current structures when the islands are not perpendicular to the shore effect upon the intensity of the up- and down-welling processes in the immediate vicinity of the peninsula.

\section{b. Island and currents in the channel separating it from} shore The influence of a near-shore island on the threedimensional current structure was studied with the configurations displayed in Fig. 16; the separation of the island from the south shore was two grid widths, i.e. $320 \mathrm{~m}$. The channel depth varied from $10 \mathrm{~m}$ at the south to $14 \mathrm{~m}$ at the north. This is more than in realistic situations for Alpine lakes.

If the channel is narrow the total mass flux through it is too small and cannot sufficiently influence the currents in the main basin, so that the current patterns obtained with the peninsula of the same effective length will describe the flow for these situations. The following peculiarities are nevertheless worth mentioning:

1. For winds from the west the currents in the channel are the largest ones in the entire basin.

2. For both winds in the long direction of the basin the very existence of the channel intensifies the current: for inflow from the west under west winds the entering current of $5 \mathrm{~cm} \mathrm{~s}^{-1}$ is enlarged in the channel interior by $2 \mathrm{~cm} \mathrm{~s}^{-1}$, for westward flow under easterly winds the entry current of $4.5 \mathrm{~cm} \mathrm{~s}^{-1}$ is enlarged by $1 \mathrm{~cm} \mathrm{~s}^{-1}$.

3. For winds from the north the along-channel velocity is small with a westward component of $0.6 \mathrm{~cm} \mathrm{~s}^{-1}$. This current is caused by the total westward movement of the upper layer in the basin (Fig. 6).

4. For winds from the south only a slight westward current of $0.05 \mathrm{~cm} \mathrm{~s}^{-1}$ is set up in the channel.

5. For winds from both north and south a divergent flow arises at the entrance of the channel.

Simulations also indicate a strong dependence of the total water flux through the channel upon the wind intensity and direction. For our wind scenarios $\left(4.7 \mathrm{~m} \mathrm{~s}^{-1}\right.$ wind speed) the flux of water through the channel for an island oriented perpendicularly to the shore is $270 \mathrm{~m}^{3} \mathrm{~s}^{-1}$ (eastward) and $200 \mathrm{~m}^{3} \mathrm{~s}^{-1}$ (westward) for winds from the west and east, and $20 \mathrm{~m}^{3} \mathrm{~s}^{-1}$ (westward) and $4 \mathrm{~m}^{3} \mathrm{~s}^{-1}$ (westward) for winds from north and south, respectively. For a weaker wind with 
the wind speed of $3 \mathrm{~m} \mathrm{~s}^{-1}$ the flux of water through the channel decreases to 120 (eastward), 102 (westward), 6 (westward) and $1 \mathrm{~m}^{3} \mathrm{~s}^{-1}$ (eastward) for winds from west, east, north and south, respectively. On the other hand, if the channel width is reduced by half and the wind speed remains unchanged $\left(4.7 \mathrm{~m} \mathrm{~s}^{-1}\right)$, the flux of water through the channel decreases to 103 (eastward), 67 (westward), 6 (westward) and $2 \mathrm{~m}^{3} \mathrm{~s}^{-1}$ (westward) for winds from west, east, north and south, respectively.

\section{Discussion and concluding remarks}

We have studied using a three-dimensional numerical code, the steady barotropic currents in a rectangular oblong basin with a coastal obstruction and variable non-symmetric bottom topography, subjected to constant and uniform winds from the four main directions. The basin geometry was varied by "inserting" a peninsula or near-shore island of different lengths in the middle of the shallower of the two long shores. To emphasise the influence of the coastal obstruction the bathymetry chosen was relatively simple, only varying across the width of the basin, asymmetrically such that the shore without peninsulas or islands would be steep and the other shore shallow. The effect of the obstructing peninsula or island was studied with regard to its efficiency separating the hydrodynamics of the subbasins formed by the obstruction and water-exchange between them. Without going into details already discussed the general results may be summarised as follows:

The least effect exercised by the peninsula on the global current distribution is obtained for winds blowing across the basin from north or south, the velocity distribution is perturbed only in the vicinity of the peninsula, and no significant global effects are induced by the obstruction. The exchange rate of the water mass through the minimum cross section between the front of the peninsula and the northern shore point is a nearly perfect linear relation between the volume flux $\left(\mathrm{m}^{3} \mathrm{~s}^{-1}\right)$ and the passing cross-sectional area $\left(\mathrm{m}^{2}\right)$. Thus the average velocity of the flow through this cross section is nearly constant (with a maximum variation of $6 \%$ ) irrespective of the size of the peninsula.

Whilst winds across the width of the basin do not generate new current structures when obstructing peninsulas are introduced this is not so for wind events in the long direction of the basin. It was found that the transition from the one-basin behaviour to a two-basin circulation with nearly decoupled individual responses is different for the two axial winds. It depends upon how much larger the area is that is blocked by the peninsula, in comparison to the total cross-sectional area of the basin.

For axial winds with the island to the left the amount of exchange water is a monotonically decreasing function of the minimum cross-sectional area. At first, when the coverage by the peninsula is $6 \%$ of the total area the drop in exchange rate is $37 \%$ followed by another $30 \%$ for the next $12 \%$. This rapid reduction for small but growing size of the peninsula is due mainly to (1) blocking the intense along-coastal jet and (2) a rearrangement of the current structure by the presence of the peninsula which is completed once about $20 \%$ of the original cross-sectional area is closed by the peninsula. A further increase of the length of the peninsula will then lead to a nearly linear decrease of the exchange water rate between the two sub-basins formed that is linearly decreasing with the passing area of the minimum cross section.

For axial winds with the island to the right the exchange rate of water between the two basins is qualitatively different even though the trend is similar. For small peninsulas covering less than $14 \%$ of the area this exchange rate remains nearly constant at a maximum (there is a negligible drop of $2 \%$ ). For a further area reduction from $14 \%$ to $33 \%$ a sharp decrease of the exchange rate of water to $34-37 \%$ from its initial value arises, which occurs because of the cyclonic gyre that is formed immediately to the west of the peninsula and extends over the entire depth of the basin. This essentially decouples the circulation in the two subbasins each of which now shows a current pattern which resembles that of the unobstructed original basin. After this subdivision into two sub-basins the exchange rate between the two sub-basins is a linear function of the passing cross-sectional area during its further reduction.

We have also scrutinised the conditions under which a depth integrated model would sufficiently accurately predict the water exchange rate between the two subbasins. That kind of model is widely used as a tool for water management, in which most tasks on pollution scenarios impact assessment are usually reduced to the evaluation of water exchange fluxes. For axial winds a two dimensional model would predict this exchange adequately, as long as the length of the obstructing peninsula is sub-critical and the re-orientation of the currents does not generate a gyre to the west of the peninsula. When the area that is blocked by the peninsula exceeds 15 to $20 \%$ of the total cross-sectional area, the exchange flux determined by the two-dimensional model is inaccurate: at first near the front of the peninsula (error of $10-12 \%$ ), and then in the entire passing area, once the remaining open area has fallen below $20 \%$ of total cross-sectional area. More significantly, and unsurprisingly, the two-dimensional results obtained for lateral winds are shown to be completely wrong, and a three-dimensional model computation is necessary for all these configurations.

When the obstruction is an island near the shore forming a small channel between island and shore, the global circulation pattern is only very slightly changed by the flow of water through the channel (of depth of $10 \mathrm{~m}$ and width of $320 \mathrm{~m}$ in our case) in comparison with the peninsula of the same length. The flux through the channel is of the order of $250 \mathrm{~m}^{3} \mathrm{~s}^{-1}$ for axial winds and at most $20 \mathrm{~m}^{3} \mathrm{~s}^{-1}$ for lateral winds. Because of the topographical narrowing, the channel intensifies the along-shore currents, resulting in an increase of the current between $25 \%$ and $40 \%$ at the channel midpoints relative to the area before the channel entrance. 
The most conspicuous effect, manifested for peninsulas with inclined axes relative to the shore, are (1) no qualitative change of the global circulation pattern when compared to the cases with peninsula axes perpendicular to the shore but (2) an intensification of the up-welling and down-welling processes very close to the peninsula in comparison to those arising for the peninsula with axis perpendicular to the shore. When the wind is directed across the basin these intensified up-welling regions lie at that shore segment of the peninsula which is to the left of the wind. The down-welling, which for winds of three directions (western, eastern and northern) occurs at the bottom slope to the east of the peninsula and was about two to three times larger if the axis of the peninsula was inclined to the east.

A final remark concerns the anisotropy of the results, which prevails through all the solutions constructed in the previous sections. This non-symmetry is due to the non-symmetric distribution of the bathymetric profile and its coupling with the rotational effects. This is responsible for the slightly but visibly different current patterns for axial and lateral winds, respectively as so already displayed in the velocity plots of Figs. 4-7. If a symmetric bathymetric profile is used rotation of any wind scenario by $180^{\circ}$ should produce current patterns that are also obtained by a rotation of $180^{\circ}$ of the currents of the original results. In Wang (1996) the circulations in a rectangular basin with a constant depth as well as with a symmetric bathymetry were studied. The results showed that in basins with constant depths no gyre occurred, as already demonstrated in subsect: 4.1, while in the basins with symmetric bathymetry the gyres were symmetric. Comparison of three solutions: of flow in a rectangular box basin, for a centrally symmetric cap-type bathymetry and for cross-asymmetric channel bathymetry, shows that (1) gyre structures appear only in the basin with topography variations, (2) symmetric topography leads to symmetric gyre structure (two gyres at opposite ends of the basin, Wang, 1996), (3) asymmetry in topography variations tends to generate asymmetric vortex structures (see Figs. 6, 7), and (4) asymmetric action of the Coriolis force generates the asymmetry in the characteristics of the vortex (Gill, 1982): cyclonic rotation (counter-clock-wise in the Northern Hemisphere) is supported and localised by Coriolis force while anti-cyclonic rotation is broken and spread upon the basin. Asymmetry in the geometry of the coastal obstruction enlarges asymmetric peculiarities in the solution. All this proves how significant asymmetries are in the interpretation of the wind-induced barotropic response of closed water basins.

Future studies will apply these findings to analyses of real basins with peninsulas or islands. Similarly, the baroclinic response will be dealt with elsewhere.
Acknowledgements. We thank Villy Kourafalou, National Center for Marine Research, Athens, Greece and the other anonymous referee for their thorough reviews of an earlier version of this paper. The new text has truly gained from their critiques.

Topical Editor N. Pinardi thanks V. Kourafalou and D. Prandle for their help in evaluating this paper.

\section{References}

Bäuerle, E., Transverse baroclinic oscillations in Lake Überlingen. Arch. Hydrobiol., 32, 409-433, 1994.

Bäuerle, E., D. Ollinger, and J. Ilmberger, Some meteorological, hydrological, and hydrodynamic aspects of Upper Lake Constance, in Arch. Hydrobiol. Spec. Issues Advan. Limnol. 53, Lake Constance, characterization of an ecosystem in transition, 31-83, 1998.

Bartholomew, J., (ed.), The Times Atlas of the World, (1955-1959), The Times Publishing Company Ltd., London, 1955-1959.

Csanady, G. T., Circulation in the coastal ocean. D. Reidel, Dordrecht, pp. 279, 1984.

Gill, A. E., Atmosphere-ocean dynamics, vol. 1, Academic Press. San Diego, California, 1982.

Haidvogel, D. B., J. L. Wilkin, and R. Young, A semi-spectral primitive equation ocean circulation model using vertical sigma and orthogonal curvilinear horizontal coordinates, J. Comput. Phys., 94, 151-185, 1991.

Heaps, N. S., Vertical structure of current in homogeneous and stratified waters, in Hydrodynamics of lakes, CISM-Lectures (Ed.) K. Hutter, Springer, Vienna-New York, 1984.

Heinz, G., J. Ilmberger, and M. Schimmele, Vertical mixing in Überlinger See, western part of Lake Constance, Aquat. Sci, 52, 256-268, 1990.

Hollan, E., and T. J. Simons, Wind-induced changes of temperature and currents in Lake Constance, Arch. Met. Geophs. Biokl., 27A, 333-373, 1978.

Hollan, E., P. F. Hamblin, and H. Lehn, Long-term modelling of stratification in large lakes: application to Lake Constance, in (Eds.) Tilzer, M.M, and Serruya, C. Large lakes - ecological structure and function, Springer, Berlin Heidelberg New York, 107-124, 1990.

Hutter, K., Fundamental equations and approximations, in Hydrodynamics of lakes, CISM-Lectures (Ed.) K. Hutter, Springer, Vienna-New York, 1-34, 1984.

Hutter, K., and Y. Wang, The role of advection and stratification in wind-driven diffusion problems of Alpine lakes, J. Lake Sci., 10, Suppl., 469-498, 1998.

Hutter, K., G. Bauer, Y. Wang, and P. Güting, Forced motion response in enclosed lakes, Coastal Est. Stud., 54, 137-166, 1998.

Kocsis, O., B. Mathis, M. Gloor, M. Schurter, and A. Wuest, Enhanced mixing in narrows: a case study at the Mainau sill (Lake Constance), Aqua. Sci., 60, 236-252, 1998.

Stocker, T., and K. Hutter, Topographic waves in channels and lakes on the f-plane, Springer, Berlin Heidelberg New York, 1987.

Wang, Y., Windgetriebene Strömungen in einem Rechteckbecken und im Bodensee, Shaker, Aachen. ISBN 3-8265-1381-9, 1996.

Wang, Y., and K. Hutter, A semi-implicit semi-spectral primitive equation model for lake circulation dynamics and its stability performance, J. Comput. Phys., 139, 209-241, 1998

Wang, Y., and K. Hutter, Methods of substructuring in lake circulation dynamics, Adv. Water Resources, 23, 399-425, 2000. 